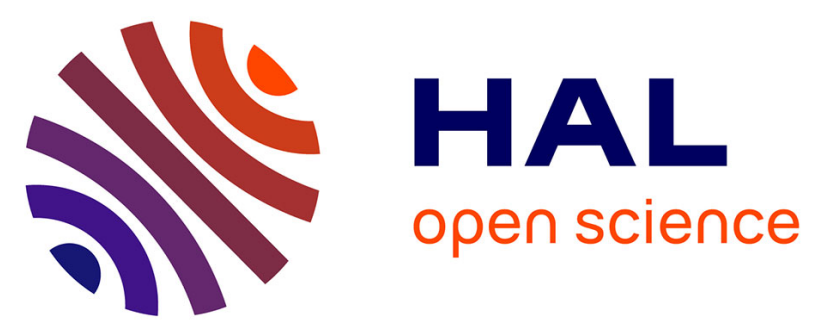

\title{
The analogous and opposing roles of double-stranded RNA-binding proteins in bacterial resistance
}

Gah-Hyun Lim, Shifeng Zhu, Kai Zhang, Timothy Hoey, Jean-Marc Deragon, Aardra Kachroo, Pradeep Kachroo

\section{- To cite this version:}

Gah-Hyun Lim, Shifeng Zhu, Kai Zhang, Timothy Hoey, Jean-Marc Deragon, et al.. The analogous and opposing roles of double-stranded RNA-binding proteins in bacterial resistance. Journal of Experimental Botany, 2019, 70 (5), pp.1627-1638. 10.1093/jxb/erz019 . hal-02119200

\section{HAL Id: hal-02119200 \\ https://hal-univ-perp.archives-ouvertes.fr/hal-02119200}

Submitted on 20 Dec 2019

HAL is a multi-disciplinary open access archive for the deposit and dissemination of scientific research documents, whether they are published or not. The documents may come from teaching and research institutions in France or abroad, or from public or private research centers.
L'archive ouverte pluridisciplinaire HAL, est destinée au dépôt et à la diffusion de documents scientifiques de niveau recherche, publiés ou non, émanant des établissements d'enseignement et de recherche français ou étrangers, des laboratoires publics ou privés. 
The analogous and opposing roles of double-stranded RNA-binding proteins in bacterial resistance

Gah-Hyun Lim ${ }^{1, a}$, Shifeng Zhu' ${ }^{1}$, Kai Zhang ${ }^{1,2}$, Timothy Hoey ${ }^{1}$, Jean-Marc Deragon $^{3}$, Aardra Kachroo ${ }^{1}$, Pradeep Kachroo ${ }^{1, *}$

${ }^{1}$ Department of Plant Pathology, University of Kentucky, Lexington, KY 40546

${ }^{2}$ College of Agronomy and Biotechnology, Southwest University, , China

${ }^{3}$ Université de Perpignan Via Domitia, CNRS UMR 5096 LGDP, 66860 Perpignan Cedex 11, Francehongqing-400716

a FarmHannong 39-23, Dongan-ro 1113ben-gil, Yeonmu-eup, Nonsansi, Chungcheongnam-do 33010, Korea

*To whom correspondencend requests for materials should be addressed.

E. mail: pk62@uky.edu

Fax: (859) 323-1961

Running Title: Role of DRB protein in bacterial resistance 


\begin{abstract}
The plasma membrane-localized plant resistance (R) protein RPM1 is degraded upon the induction of the hypersensitive response (HR) triggered in response to its own activation or that of other unrelated R proteins. We tested a role for RPM1 turnover in RPM1-mediated resistance and show that degradation of RPM1 is not associated with HR or resistance mediated by this R protein. Likewise, the runaway cell death phenotype in $l s d 1$ mutant was not associated with RPM1 degradation and did not alter RPM1-derived resistance.RPM1 stability and RPM1-mediated resistance were dependent on the double-stranded RNA binding (DRB) proteins 1 and 4. Interestingly, the function of DRB1 in RPM1-mediated resistance was not associated with its role in pre-miRNA processing. The DRB3 and DRB5 proteins negatively regulated RPM1 mediated resistance and a mutation in these completely or partially restored resistance in the $d r b 1, d r b 2$ and $d r b 4$ mutant backgrounds. Conversely, plants overexpressing DRB5 showed attenuated RPM1-mediated resistance. A similar role for DRBs in basal and R-mediated resistance suggest that these proteins play a general role in bacterial resistance.
\end{abstract}




\section{Introduction}

Plants utilize active defense mechanisms such as specific induction of elaborate defense signaling pathways to counter microbes. The various modes of defenses induced upon the recognition of pathogen-derived molecules provide local resistance to race-specific pathogens, and basal resistance to virulent pathogens. Resistance $(R)$ gene-mediated or species-specific immunity (also termed EffectorTriggered Immunity: ETI) is induced when a strain-specific effector or avirulence protein (AVR) from the pathogen associates directly/indirectly with the cognate plant R protein [reviewed in (Kachroo 2006; Jones and Dangl 2006; Chisholm et al. 2006)]. Some R proteins perceive the presence of the pathogen via direct physical interactions with the cognate Avr proteins (Scofield et al. 1996; Jia et al. 2000; Todd and Fumiaki 2000; Deslandes et al. 2003), but the majority of R proteins associate indirectly with cognate Avr proteins. A well-studied example of an indirect mode of effector recognition is that of the Arabidopsis (Arabidopsis thaliana) $\mathrm{R}$ protein, RPM1 (for resistance to Pseudomonas syringae pv maculicola 1). RPM1 mediates resistance against bacteria expressing two different Avr proteins, AvrRpm1 and AvrB. Although RPM1 does not directly interact with either AvrRpm1 or AvrB, it does associate with RIN4 (for RPM1-interacting 4), which interacts with AvrRpm1 and AvrB. RIN4 is required for RPM1-induced resistance to AvrRpm1/AvrBexpressing P. syringae pv. tomato (Pst) (Mackey et al. 2002). Both AvrRpm1 and AvrB induce the phosphorylation of RIN4, which is associated with RPM1 activation and the resulting resistance signaling.

$\mathrm{R}$ proteins are thought to transition from an inactive to active state upon direct or indirect binding to the AVR protein. The dormant state of RPM1 is stabilized by RAR1 (required for Mla12-mediated resistance) and DRB4 (doublestranded RNA binding protein 4) (Schulze-Lefert 2004; Zhu et al. 2013). RAR1 and DRB4 likely regulate RPM1 stability via independent processes because $d r b 4$ mutant plants contain wild-type-like levels of RAR1. The Arabidopsis genome encodes four other DRB isoforms, which have been primarily studied for their roles in RNA silencing. Of these, DRB1 and DRB4 facilitate DCL1 (Dicer like 1)- and DCL4mediated synthesis of miRNA and trans-acting siRNAs (tasiRNAs), respectively (Vazquez et al. 2004; Adenot et al. 2006). DRB2 is also involved in the biogenesis of specific miRNA subsets (Eamens et al. 2012a) and DRB3 and DRB5 are thought to function in the same non-canonical miRNA pathway as DRB2 (Eamens et al. 2012a). Interestingly, all DRB isoforms contribute to the stability of the R protein HRT (HR to Turnip Crinkle Virus), which confers resistance against turnip crinkle virus (TCV). In addition to stabilizing HRT, DRB1 also plays a role in HRT activation because a mutation in DRB1 compromises HR to TCV. The DRB1 and DRB4 proteins are positively regulated by COP1 (Cho et al. 2014; Lim et al. 2018), an E3 ubiquitin ligase that functions as a negative regulator of photomorphogenesis (Lau and Deng 
2012). Thus, a mutation in COP1 affects the stability of both HRT and RPM1 (Lim et al. 2018). Consequently, the cop1 mutant shows pronounced susceptibility to Pst avrRpm1.

Induction of R-mediated responses is often accompanied by the formation of a hypersensitive reaction (HR), a form of programmed cell death resulting in necrotic lesions, at the site of pathogen entry (Dangl et al. 1996). HR is one of the first visible manifestations of pathogen-induced host defenses. Interestingly, RPM1 is degraded in response to HR induced by Pst avrRpm1 or the RPS2 (resistance to Pst 2)-mediated recognition of Pst avrRpt2 (Boyes et al. 1998). This led to the suggestion that RPM1 turnover regulates the extent of cell death and the amplitude of resistance response at the site of infection (Boyes et al. 1998).

Here, we examined the roles of HR and DRB proteins in RPM1-mediated resistance against Pst avrRpm1. We show that degradation of RPM1 post pathogen infection is not associated with RPM1-mediated HR or the resistance response. Unlike their roles in the HRT-TCV pathosystem, DRB3 and DRB5 proteins negatively regulate defense against bacteria, and of these DRB5 negatively regulates the function of DRB2 in basal- and R-mediated resistance. We also show that DRB1 and DRB4 are required for RPM1 stability. Together, our results show that although some DRB isoforms have identical functions, others have more distinct roles in defense against bacterial and viral pathogens.

\section{Results}

\section{DRB1, DRB2 and DRB4 proteins are required for RPM1-mediated resistance}

The Arabidopsis genome encodes five DRB proteins and all contribute to HRT-mediated resistance against TCV (Lim et al., 2018). Of these, DRB4 is required for RPM1 stability and thereby RPM1-mediated resistance (Zhu et al., 2013). Based on the fact that HRT and RPM1 are both peripheral plasma membrane-localized proteins (Jeong et al. 2010; Boyes et al. 1998), we considered the possibility that DRB proteins function similarly in defense derived from these two R proteins. To test this, we analyzed DRB protein levels in response to avrRpm1 infection in wildtype and transgenic plants expressing epitope-tagged DRB proteins in the respective $d r b$ mutant background (Table S1). The $d r b$ lines used here have been characterized in earlier studies (Curtin et al., 2008; Lim et al., 2018; Table S1). A time-course analysis of DRB levels was conducted after pathogen inoculation. Infection with Pst avrRpm1 resulted in increased accumulation of DRB1, DRB2 and DRB4 within $24 \mathrm{~h}$ of infection (Figure 1A). The DRB3 and DRB5 proteins were undetectable in both mock- and Pst avrRpm1-infected plants. This suggested that DRB1 and DRB2 may contribute to RPM1-mediated resistance, as already shown for DRB4 (Zhu et al., 

lines. Notably, $d r b 1-2, d r b 2-1$ and $d r b 4-1$ showed prominent chlorotic symptoms (S. Figure 1A) and supported $\sim 7-10$-fold higher levels of Pst avrRpm1 (Figure 1B), suggesting that these KO lines were compromised in RPM1-mediated resistance. This was not the case for $d r b 3-1$ or $d r b 5-1$ (Figure 1B, S. Figure 1A), suggesting that these DRB proteins either did not participate in resistance to Pst avrRpm1 or had other undiscernible roles. A similar trend was also seen in $d r b$ mutants infected with Pst avrRpt2 or virulent Pst (DC3000); drb1, drb2, and drb4 showed enhanced susceptibility while $d r b 3$ and $d r b 5$ showed wild-type-like resistance (S. Figures $1 \mathrm{~B}$, 1C). This suggested that DRB1, DRB2 and DRB4 proteins positively regulate resistance to Pst. Noticeably, $d r b 1$ plants showed higher susceptibility as compared to $d r b 2$ and $d r b 4$ (Figure 1B), suggesting that DRB1 had a more significant role in resistance against $P s t$. Consistent with their enhanced susceptibility phenotype, the $d r b 1, d r b 2$ and $d r b 4$ plants showed significantly less ion-leakage compared to wildtype or $d r b 3$ and $d r b 5$ plants (Figure $1 \mathrm{C}$ ). This correlated with the significantly reduced SA accumulation and $P R-1$ expression in pathogen-infected $d r b 1, d r b 2$ and $d r b 4$ plants (Figures 1D, 1E) (Zhu et al. 2013). These results indicated a role for DRB1, DRB2 and DRB4 in RPM1-mediated resistance.

The canonical functions of DRB1 and DRB2 proteins are in miRNA accumulation (Vaucheret et al. 2004; Vazquez et al. 2004; Han et al. 2004). Notably, while the full-length DRB2 is essential for miRNA biosynthesis, the N-terminal domain of DRB1 is sufficient for miRNA biosynthesis (Wu et al. 2007). We reasoned that if the role of DRB1 in RPM1-mediated resistance was associated with its function in miRNA processing, expression of the N-terminal DRB1 domain in $d r b 1$ plants ( $\triangle^{\mathrm{C}} \mathrm{DRB} 1$ in Figure 2, Wu et al. 2007) should be sufficient to rescue their defense-related defects. However, the $d r b 1:: \triangle^{C} D R B 1$ plants were as susceptible to Pst avrRpm1 or virulent Pst as $d r b 1$ plants, whereas $d r b 1:: D R B 1$ showed wild-type like resistance (Figures 2A, 2B). This was consistent with the reduced $P R-1$ expression in Pst avrRpm1 infected $d r b 1:: \triangle^{C} D R B 1$ plants (Figure $2 C$ ). In comparison, full length transgenic expression of DRB1 complemented the defense phenotypes associated with $d r b 1$ mutation (Figures 2A, 2B, 2C). Thus, the function of DRB1 in RPM1-mediated resistance did not appear to be associated with its role in premiRNA processing. We confirmed this further by evaluating RPM1-mediated resistance in dcl1 and ago1 plants, since both DCL1 and AG01 play important roles in miRNA biosynthesis (Achkar et al. 2016); the dcl1 and ago1 plants showed wildtype-like resistance to avrRpm1 Pst (Figure 2D).

\section{DRB1 regulates stability of the RPM1 protein}

DRB4 was previously shown to regulate RPM1 stability (Zhu et al., 2013) and both DRB1 and DRB2 were required for RPM1-derived resistance (Figure 1B). 
121 Therefore, we considered the possibility that the inhibition of RPM1-derived 122 resistance in $d r b 1$ and $d r b 2$ plants to Pst avrRpm1 might be associated with reduced 123 stability of the RPM1 protein. We crossed the $d r b$ mutants with transgenic plants 124 expressing RPM1-MYC under the RPM1 native promoter. The RPM1-MYC line used 125 here has been characterized in several earlier studies (Boyes et al. 1998; Kawasaki et al. 2005). At least five independent F2 plants were tested and all drb1 RPM1-MYC or drb4 RPM1-MYC plants were found to contain dramatically reduced RPM1-MYC as compared to wild-type plants (Figure 3A). The reduced RPM1 protein in these plants was likely a post-transcriptional response because $d r b 1$ and $d r b 4$ plants expressed wild-type like levels of RPM1-MYC transgene (S. Figure 2A). Unlike $d r b 1$ and $d r b 4$ mutants, increased susceptibility seen in $d r b 2$ was not associated with RPM1-MYC levels; the $d r b 2$ plants contained wild-type-like levels of RPM1-MYC (Figure 3A). Notably, despite their reduced RPM1 levels the $d r b 1$ and $d r b 4$ plants showed better resistance compared to rpm1 plants suggesting that the RPM1 protein was not completely absent in these backgrounds (Figure 3B). We reasoned that if $d r b 1$ and $d r b 4$ plants contained at least some RPM1 protein it should be possible to restore Pst avrRpm1 resistance in these plants by compensating for a downstream factor such as SA. Indeed, avrRpm1 infection increased SA levels in wild-type plants (Figure 1D) and impaired biosynthesis of SA in sid2 plants (which are unable to synthesize pathogen-responsive SA; Wildermuth et al., 2001) conferred enhanced susceptibility to Pst avrRpm1 (Figure 3C). Furthermore, similar expression of $P R-1$ was detected in plants treated with SA, Pst avrRpm1 or SA+ Pst avrRpm1 (S. Figure 2B). Together, these results suggest that SA operates downstream of RPM1. Consistent with this notion, treatment with SA induced wildtype-like $P R-1$ expression in $d r b$ plants (S. Figure 2C) and enhanced resistance of $d r b 1, d r b 2$ and $d r b 4$ plants to Pst avrRpm1 (Figure 3C). Likewise, SA pretreatment enhanced resistance of sid2 plants to Pst avrRpm1 (Figure 3C).

Activation of ETI is often associated with the induction of the systemic acquired resistance (SAR) response and this is dependent on the presence of the $\mathrm{R}$ protein and the SA pathway (Gao et al. 2014). We tested the SAR response of $d r b 1$, $d r b 2$ and $d r b 4$ plants, which contained reduced RPM1 protein or showed reduced accumulation of SA (Figures 1D, 3A). Wild-type (Col-0) and $d r b$ plants were inoculated with $\mathrm{MgCl}_{2}$ or Pst avrRpm1 and $48 \mathrm{~h}$ later the distal leaves of all plants were challenged with virulent Pst (Pst Vir). The growth of Pst Vir was monitored at 0 and 3 dpi. As expected, wild-type plants induced SAR; pre-exposure to Pst avrRpm1 resulted in $\sim 10$-fold reduction in the growth of the secondary pathogen Pst Vir (Figure 3D). This was also the case for the $d r b 3$ and $d r b 5$ mutants. In contrast, $d r b 1$, $d r b 2$ and $d r b 4$ mutant lines did not induce SAR. Together, these results indicate that, DRB1, DRB2 and DRB4 proteins contribute to RPM1-mediated downstream signaling leading to the establishment of SAR. 


\section{RPM1 turnover does not contribute to RPM1-mediated signaling}

RPM1 is rapidly degraded after the onset of HR in response to pathogen infection and this is considered to feed-back regulate HR-associated cell death and the overall resistance response (Boyes et al. 1998). However, turnover of proteins involved in a signaling response can also play a regulatory role in physiological processes (Spoel et al. 2009; Zuo et al. 2012). This prompted us to examine whether turnover of RPM1 was required for RPM1-mediated resistance by evaluating the role of HR-associated cell death in RPM1 turnover and RPM1-derived resistance.

First, we examined whether HR-associated RPM1 degradation was derived from protease activity or the 26S proteasome. The rpm1 RPM1-MYC plants (RPM1MYC expressed under the RPM1 native promoter) were treated with a protease inhibitor, the 26S proteasome-specific inhibitor MG132, or the solvent DMSO (as control). Infection with Pst avrRpm1 resulted in RPM1-MYC degradation within $1 \mathrm{~h}$ in plants pretreated with DMSO or the protease-specific inhibitor, but not in plants pretreated with MG132 (Figure 4A). However, all treated plants exhibited significant ion-leakage in response to pathogen infection (data shown for MG132, Figure $4 \mathrm{~B}$ ). This suggested that the $26 \mathrm{~S}$ proteasome was responsible for RPM1 turnover in response to pathogen-induced HR and that turnover of RPM1 was not essential for proper onset of HR. RPM1 turnover was also not essential for subsequent resistance signaling because the protease inhibitor- and MG132-treated plants exhibited similar $P R-1$ induction and Pst avrRpm1 resistance as the control plants (Figures 4C, 4D).

Next, we assayed RPM1 levels in lsd1 RPM1-MYC plants. The lsd1 mutant exhibits runaway cell death phenotype in response to biotic and abiotic stresses (Dietrich et al. 1997; Mateo et al. 2004). Consistent with previous reports for lsd1 (Xiaozhen et al. 2010), lsd1 RPM1-MYC plants grown in long day conditions (14 h day light), showed photo-oxidative stress and accumulated increased levels of reactive oxygen species (ROS), which was highly elevated after pathogen inoculation (S. Figure 3A). These plants contained wild-type-like levels of RPM1-MYC (Figure 4E). We next assayed RPM1-MYC levels after avrRpm1 inoculation. As shown before, RPM1-MYC was degraded within $3 \mathrm{~h}$ of avrRpm1 inoculation in the wild-type background (Figure 4F). In contrast, pathogen infection did not noticeably reduce RPM1-MYC protein levels in the lsd1 background, even though these plants showed pronounced cell death (S. Figure 3B). These results suggested that avrRpm1-induced degradation of RPM1 is not merely a factor of the cell death response. It is possible that a more controlled HR is required for RPM1 turnover. Alternatively, increased accumulation of one or more defense compounds in the ls 1 background may result in increased stability of the RPM1 protein in these plants. Consistent with earlier observations (Xiaozhen et al. 2010), the lsd1 RPM1-MYC plants showed increased accumulation of 
ROS (S. Figure 3A) and elevated RPM1 transcript levels (Figure 4G). Moreover, the

202 Isd1 plants showed normal resistance to avrRpm1 (Rustérucci et al. 2001). Together, 203 these data indicate that HR-associated turnover of RPM1 is not essential for RPM1204 mediated pathogen resistance. Thus, increased susceptibility in $d r b 1$ and $d r b 4$

\section{DRB3 and DRB5 act as negative regulators of RPM1-mediated resistance}

Unlike DRB1 or 4, the DRB3 and DRB5 isoforms did not appear to influence bacterial resistance or RPM1 protein stability (Figures 1B, 3A). One possibility was that DRB3 and DRB5 were redundant in their functions at least for bacterial resistance, because these isoforms do contribute individually to viral resistance (Lim et al. 2018). We tested their potential functional redundancy by generating $d r b 3 d r b 5$ double mutant plants and evaluating their response to Pst (S. Figures 4A, 4B; Figures 5A, 5B, 5C). Interestingly, the $d r b 3 d r b 5$ plants showed slightly enhanced resistance to virulent Pst (Figures 5B, S4A) and wild-type-like resistance to Pst avrRpm1 (Figures 5C, S4B). This suggested that the functional redundancy between $D R B 3$ and $D R B 5$ proteins was limited to their additive effect on basal resistance.

Considering their opposite effects on bacterial resistance, we next generated double mutant combinations to evaluate epistasis between $d r b 3$ or $d r b 5$ and $d r b 1$, $d r b 2$, or $d r b 4$ (Figure $5 \mathrm{~A}$ ). Interestingly, the $d r b 5$ mutation restored basal resistance in $d r b 1$ and $d r b 2$ plants (Figure 5B). In contrast, the $d r b 3$ mutation restored basal resistance in the $d r b 4$ background but did not alter the response of $d r b 2$ plants (Figure 5B). The $d r b 5$ mutation also completely or partially restored resistance to Pst avrRpm1 in $d r b 1$ and $d r b 2$ plants, respectively, but did not alter the resistance response of $d r b 4$ plants (Figure $5 \mathrm{C}$ ). In contrast, the $d r b 3$ mutation partially restored Pst avrRpm1 resistance in $d r b 1$, but not in $d r b 2$ or $d r b 4$ plants (Figure 5C). Together, these data suggest that the DRB5 negatively regulates the functions of the DRB1 and DRB2 proteins during both basal and $R$-mediated resistance to Pst (Figure 5D). In comparison, DRB3 negatively regulated the function of DRB1 during both basal and $R$-mediated resistance to Pst and that of DRB4 in basal resistance (Figure 5D).

We next tested if $D R B 3$ or DRB5 overexpression altered the RPM1-mediated resistance response in the wild-type (Col-0) background. Transgenic plants overexpressing $D R B 3$ or $D R B 5$ were screened based on their transcript levels 
(Figures 6A, 6B) and at least two independent transgenic lines each were evaluated for their response to Pst avrRpm1. The DRB3- and DRB5-overexpressing plants showed wild-type-like morphology (Figure 6A), and of these $35 S$-DRB5 plants

\section{Discussion} consistently exhibited enhanced susceptibility to Pst avrRpm1 (Figure 6C). The DRB5 overexpressing plants also showed enhanced susceptibility to virulent $P s t$ as well as Pst avrRpt2 (S. Figure 5). Their increased susceptibility correlated with reduced ion-leakage in response to pathogen infection (Figure 6D). The DRB3 overexpressing plants showed a nominal reduction in ion-leakage but in most experiments these plants showed wild-type-like resistance to avrRpm1, avrRpt2 or virulent Pst (Figures 6C, S5). Together, these results showed that DRB5-mediated negative regulation had an effect on bacterial resistance.

\section{Discussion}

In this study, we evaluated five DRB isoforms, which are known to participate in miRNA and siRNA biogenesis, for their roles in basal and R-mediated resistance to $P$. syringae. Our results show that the various DRB isoforms have specific and unique functions in bacterial defense; DRB1, DRB2 and DRB4 isoforms function as non-redundant positive regulators of resistance, while DRB3 and DRB5 operate as negative regulators. The DRB2, DRB3 and DRB5 proteins are thought to function in the same non-canonical pathway during miRNA biogenesis (Eamens et al. 2012b). However, their differing functions in bacterial resistance suggests that the functions of DRB proteins in defense signaling may not strictly overlap with their functions in the miRNA pathway. This is further corroborated by the fact that the $\mathrm{C}$-terminal truncated form of DRB1, which is functional in miRNA biogenesis is unable to complement resistance signaling in the $d r b 1$ mutant background (Wu et al. 2007; Yang et al. 2010). The C-terminal truncated DRB1 lacks the nuclear localization signal (NLS) and protein-protein interaction (PPI) domains (Wu et al. 2007). Although neither the NLS or PPI domains are required for miRNA processing, these regions are clearly important for DRB1-mediated defense against bacteria.

The roles of at least some of the DRB isoforms in plant defense appears to be associated with their ability to regulate R protein stability, which in turn could be mediated through direct or indirect interactions with the R protein. For instance, the DRB1 and DRB4 proteins regulate viral resistance because they regulate the stability of the R protein HRT (Lim et al. 2018). However, the DRB2 isoform which also contributes to HRT stability and HRT-mediated resistance does not directly interact with HRT. Notably, unlike their role in HRT-mediated resistance, DRB2 is not required for the stability of RPM1. This suggests that DRB2 likely functions at a downstream step in RPM1-mediated resistance. 
Our results show that the various DRB proteins have differing functions in defense against $P$ st versus TCV. For instance, DRB2 functions as a positive regulator of defense against both Pst and TCV, whereas DRB3 and DRB5 negatively regulate defense against $P s t$, and positively regulate HRT-mediated resistance to TCV. The similar functions of DRB3 and DRB5 in defense against Pst are consistent with their common involvement in the non-canonical miRNA pathway (Eamens et al. 2012a). However, DRB3 and DRB5 have also been suggested to function in the DRB2dependent miRNA pathway (Eamens et al. 2012a), but DRB2 and DRB3/DRB5 have opposing functions in bacterial defense. DRB3 was recently shown to regulate basal resistance to geminiviruses via its effect on changes to chromatin methylation (Raja et al. 2014). This suggests that DRB3 could contribute to bacterial resistance by regulating chromatin methylation, which are well known to play a role in bacterial resistance (Alexandre et al. 2012).

Both DRB2 and DRB4 proteins are part of a higher molecular weight complex (Clavel et al. 2015) and DRB2 interacts with proteins involved in the regulation of chromatin function (Clavel et al. 2015). Notably, the high molecular weight DRB2 complex contains MSI4, which functions as a substrate adaptor for CULLIN4 (CUL4)Damaged DNA Binding Protein1 (DDB1) ubiquitin E3 ligases. CULLIN4 E3 ubiquitin ligases in turn interact with COP1, an important negative regulator of photomorphogenesis and a positive regulator of RPM1-mediated resistance. COP1 regulates RPM1 resistance by stabilizing DRB1 and DRB4 which are required for RPM1 stability (Cho et al. 2014; Lim et al. 2018). Thus, like the $d r b 1$ and $d r b 4$ mutants, the cop1 mutant also contains little to no RPM1 protein and exhibits enhanced susceptibility to Pst (Lim et al. 2018).

In addition to COP1, RPM1 also interacts with two membrane bound E3 ligases RIN2 and RIN3. Although RIN2 and RIN3 positively regulate RPM1-triggered HR, they do not contribute to RPM1-mediated resistance (Tsutomu et al. 2005). RPM1 is unique in that it is the only known R protein that is degraded upon induction of HR (Boyes et al. 1998). COP1 is unlikely to contribute to this HR associated degradation of RPM1 because it is a positive regulator of RPM1-mediated resistance. Furthermore, mutations in RIN2/RIN3 has no effect on RPM1 level, suggesting that RIN2/RIN3 E3 ligases are not involved in pathogen-triggered degradation of RPM1 (Tsutomu et al. 2005). This is consistent with our results that show HR associated degradation of RPM1 is not essential for RPM1-derived resistance. This is based on our finding that $26 \mathrm{~S}$ proteasome inhibitor MG132 inhibited RPM1 degradation but did not alter RPM1-mediated HR or resistance. In addition, RPM1 was not degraded in $l s d 1$ plants, which showed pronounced pathogen-induced cell death. It is possible that lsd1 plants show normal resistance despite their lack of RPM1 turnover because they contain increased levels of ROS 
(Dietrich et al., 1997; Mateo et al., 2004) or other defense-associated molecules. This was consistent with the observation that exogenous application of glycerol resulted in induction of RPM1-MYC transcript and conferred enhanced resistance to virulent Pst (Venugopal et al. 2009).

Notably, overexpression of the DRB5 protein compromised RPM1-mediated resistance to avrRpm1 Pst but did not affect HRT-mediated resistance to TCV. This together with the shared dependence of HRT- and RPM1-mediated resistance on SA suggests that DRB3 and DRB5 proteins might not influence defense by perturbing $S A$ levels. Indeed, mutation or overexpression of $D R B 3$ or $D R B 5$ does not alter levels of basal or pathogen induced SA. However, overexpression of DRB3 or DRB5 did lower pathogen-induced ion-leakage with 35S-DRB5 plants showing more pronounced reduction. Thus, it is possible that DRB3 and DRB5 proteins negatively regulate a subset of RPM1-derived events leading to $H R$, and this in turn regulates resistance. A nominal reduction of ion-leakage in 35S-DRB3 plant versus a more pronounced effect seen in 35S-DRB5 correlates well with their respective resistance response to Pst avrRpm1. Thus, while degradation of RPM1 is not essential for the onset of HR or resistance, loss of HR might contribute to RPM1-mediated resistance.

\section{Experimental Procedures}

\section{Plant growth conditions, genetic analysis and generation of transgenic plants}

Plants were grown in MTPS 144 Conviron (Winnipeg, MB, Canada) walk-inchambers at $22{ }^{\circ} \mathrm{C}, 65 \%$ relative humidity and 14 hour photoperiod. The photon flux density of the day period was $106.9 \mu$ moles $\mathrm{m}^{-2} \mathrm{~s}^{-1}$ and was measured using a digital light meter (Phytotronic Inc, Earth city, M0). Plants were grown on autoclaved ProMix soil (Premier Horticulture Inc., PA, USA). Soil was fertilized once using Scotts Peter's 20:10:20 peat lite special general fertilizer that contained $8.1 \%$ ammoniacal nitrogen and 11.9\% nitrate nitrogen (Scottspro.com). Plants were irrigated using deionized or tap water.

The $d r b 1-2, d r b 2-1, d r b 3-1, d r b 4-1, d r b 5-1$ genotypes used in this study are described in Table S1. Crosses were performed by emasculating the flowers of the recipient genotype and pollinating with the pollen from the donor. F2 plants showing the wild-type genotype at the mutant locus were used as controls in all experiments. The wild-type and mutant alleles were identified by PCR, CAPS, or dCAPS analysis.

For transgenic overexpression of $D R B s$, the cDNA spanning the coding region in pGWB2 vector (Nakagawa et al., 2007), which after confirmation of the DNA sequence was transformed into Col-0 plants. The transgenic plants were selected on plates containing kanamycin $(50 \mu \mathrm{g} / \mathrm{ml})$ and hygromycin $(17 \mu \mathrm{g} / \mathrm{ml})$. For native 
expression of $D R B s$, the MYC tagged DRBs along with their respective promoters were cloned into pCambia 1300 derived vector and transformed into respective $d r b$ mutant backgrounds (Clavel et al. 2015; Lim et al. 2018). Genetic complementation was assayed by analyzing the levels of siRNA, as described before (Clavel et al., 2015).

\section{RNA extraction, RNA gel-blot analyses and qRT-PCR}

Small-scale extraction of RNA from two or three leaves (per sample) was performed with the TRIzol reagent (Invitrogen, CA), following the manufacturer's instructions. RNA gel blot analysis and synthesis of random-primed probes for $P R-1$ were carried out as described previously (Kachroo et al., 2000).

RNA quality and concentration were determined by gel electrophoresis and determination of $\mathrm{A}_{260}$. Reverse transcription (RT) and first strand cDNA synthesis were carried out using Superscript II (Invitrogen, CA). Quantitative RT-PCR was carried out as described before (Zhang et al., 2009). Each sample was run in triplicates and ACTIN expression levels were used as internal control for normalization. Cycle threshold values were calculated by SDS 2.3 software. Genespecific primers used for real-time quantitative RT-PCR analyses are described in Table S2.

\section{Trypan-blue staining}

The leaves were vacuum-infiltrated with trypan-blue stain prepared in 10 $\mathrm{mL}$ acidic phenol, $10 \mathrm{~mL}$ glycerol, and $20 \mathrm{~mL}$ sterile water with $10 \mathrm{mg}$ of trypan blue. The samples were placed in a heated water bath $\left(90^{\circ} \mathrm{C}\right)$ for 2 min and incubated at room temperature for 2-12 $\mathrm{h}$. The samples were destained using chloral hydrate ( $25 \mathrm{~g} / 10 \mathrm{~mL}$ sterile water; Sigma), mounted on slides and observed for cell death with a compound microscope. The samples were photographed using an AxioCam camera (Zeiss, Germany) and images were analyzed using Openlab 3.5.2 (Improvision) software.

\section{Conductivity assays}

Electrolyte leakage was measured in four-week-old plants as described earlier (Yu et al., 2013). Briefly, leaves were infiltrated with $\mathrm{MgCl}_{2}$ or avrRpt2 Pst $\left(10^{6} \mathrm{CFU} / \mathrm{ml}\right)$ and 5 leaf discs $(7 \mathrm{~mm})$ per plant were removed with a cork borer, floated in distilled water for $50 \mathrm{~min}$, and subsequently transferred to tubes containing $5 \mathrm{ml}$ of distilled water. Conductivity of the solution was determined with an NIST traceable digital Conductivity Meter (Fisher Scientific). Standard deviation was calculated from four replicate measurements per genotype per experiment.

\section{Pathogen infections}


The bacterial strain avrRpm1 was grown overnight in King's B medium containing rifampicin and kanamycin (Sigma, M0). The bacterial cells were harvested, washed and suspended in $10 \mathrm{mM} \mathrm{MgCl}_{2}$. The cells were diluted to a final

404 density of $10^{5} \mathrm{CFU} / \mathrm{mL}\left(\mathrm{A}_{600}\right)$ and used for infiltration. The bacterial suspension was 405 injected into the abaxial surface of the leaf using a needle-less syringe. Three leaf 406 discs from the inoculated leaves were collected at 0 and 3 dpi. The leaf discs were homogenized in $10 \mathrm{mM} \mathrm{MgCl}$, diluted $10^{3}$ or $10^{4}$ fold and plated on King's B medium. For analysis of SAR, the primary leaves were inoculated with $\mathrm{MgCl}_{2}$ or the avr bacteria $\left(10^{7} \mathrm{cfu} \mathrm{\textrm {ml } ^ { - 1 } )}\right.$ and, $48 \mathrm{~h}$ later, the systemic leaves were inoculated with vir bacteria $\left(10^{5} \mathrm{cfu} \mathrm{ml}^{-1}\right)$. The samples from the systemic leaves were harvested at 3 411 dpi.

\section{Protein extraction and Immunoblot analysis}

Proteins were extracted in buffer containing $50 \mathrm{mM}$ Tris- $\mathrm{HCl}, \mathrm{pH} 7.5,10 \%$ glycerol, $150 \mathrm{mM} \mathrm{NaCl}, 10 \mathrm{mM} \mathrm{MgCl}_{2}, 5 \mathrm{mM}$ EDTA, $5 \mathrm{mM}$ DTT, and $1 \mathrm{X}$ protease inhibitor cocktail (Sigma-Aldrich, St. Louis, MO). Protein concentration was measured by the Bio-RAD protein assay (Bio-Rad, CA). For small scale extractions 23 leaves were homogenized per sample. For Ponceau-S staining, PVDF membranes were incubated in Ponceau-S solution ( $40 \%$ methanol (v/v), 15\% acetic acid (v/v), $0.25 \%$ Ponceau-S). The membranes were destained using deionized water. Proteins $(30-50 \mu \mathrm{g})$ were fractionated on a $7-10 \%$ SDS-PAGE gel and subjected to immunoblot analysis using $\alpha$-MYC (Sigma-Aldrich, St. Louis, M0) antibody. Immunoblots were developed using ECL detection kit (Roche) or alkalinephosphatase-based color detection.

425

426 


\section{Acknowledgements}

428 This work was supported by grants from the National Science Foundation 429 (IOS\#0749731, \#1457121). We thank Yuke He for drb1::DRB1 and drb1:: $\triangle^{c} D R B 1$ 430 transgenic lines, Jeff Dangl for RPM1-MYC and Arabidopsis database for DRB 431 knockout seeds. We thank Wendy Havens and Ludmila Lapchyk for technical help 432 and Amy Crume for managing the plant growth facility.

433

\section{Author contributions}

435 Genetic analysis, pathogen infections, generation of $D R B$ overexpressing plants, qRT, 436 RNA gel-blot- and immunoblot-analysis was carried out by GHL with help from SZ,

437 KZ and TH. PK supervised the project, PK and AK analyzed data, and wrote the 438 manuscript with contribution from all the authors.

439

440 Competing financial interests

441 The authors declare no competing financial interests. 


\section{References}

Achkar NP, Cambiagno DA, Manavella PA (2016) miRNA Biogenesis: A dynamic pathway. Trends in Plant Science 21 (12):1034-1044. doi:10.1016/j.tplants.2016.09.003

Adenot X, Elmayan T, Lauressergues D, Boutet S, Bouché N, Gasciolli V, Vaucheret H (2006) DRB4-dependent TAS3 trans-acting siRNAs control leaf morphology through AGO7. Current Biology 16 (9):927-932. doi:10.1016/j.cub.2006.03.035

Alexandre B, Rozenn M, Thierry H, Wen-Hui S (2012) Chromatin modification and remodelling: a regulatory landscape for the control of Arabidopsis defence responses upon pathogen attack. Cellular Microbiology 14 (6):829-839. doi:doi:10.1111/j.1462-5822.2012.01785.x

Boyes DC, Nam J, Dangl JL (1998) The Arabidopsis thaliana RPM1 disease resistance gene product is a peripheral plasma membrane protein that is degraded coincident with the hypersensitive response. Proceedings of the National Academy of Sciences of the United States of America 95 (26):15849-15854

Chisholm ST, Coaker G, Day B, Staskawicz BJ (2006) Host-microbe interactions: Shaping the evolution of the plant immune response. Cell 124 (4):803-814

Cho SK, Chaabane SB, Shah P, Poulsen CP, Yang SW (2014) COP1 E3 ligase protects HYL1 to retain microRNA biogenesis. Nature Communications 5:5867. doi:10.1038/ncomms6867

Clavel M, Pélissier T, Descombin J, Jean V, Picart C, Charbonel C, Saez-Vásquez J, Bousquet-Antonelli C, Deragon J-M (2015) Parallel action of AtDRB2 and $\mathrm{RdDM}$ in the control of transposable element expression. BMC Plant Biology 15 (1):70. doi:10.1186/s12870-015-0455-Z

Dangl JL, Dietrich RA, Richberg MH (1996) Death don't have no mercy: Cell death Programs in plant-microbe interactions. Plant Cell 8 (10):1793-1807. doi:10.1105/tpc.8.10.1793

Deslandes L, Olivier J, Peeters N, Feng DX, Khounlotham M, Boucher C, Somssich I, Genin S, Marco Y (2003) Physical interaction between RRS1-R, a protein conferring resistance to bacterial wilt, and PopP2, a type III effector targeted to the plant nucleus. Proceedings of the National Academy of Sciences 100 (13):8024-8029. doi:10.1073/pnas.1230660100

Dietrich RA, Richberg MH, Schmidt R, Dean C, Dangl JL (1997) A novel zinc finger protein is encoded by the Arabidopsis $L S D 1$ gene and functions as a negative regulator of plant cell death. Cell 88 (5):685-694

Eamens AL, Kim KW, Curtin SJ, Waterhouse PM (2012a) DRB2 is required for microRNA biogenesis in Arabidopsis thaliana. PLoS One 7. doi:10.1371/journal.pone.0035933

Eamens AL, Wook Kim K, Waterhouse PM (2012b) DRB2, DRB3 and DRB5 function in a non-canonical microRNA pathway in Arabidopsis thaliana. Plant Signaling \& Behavior 7 (10):1224-1229. doi:10.4161/psb.21518

Gao Q-m, Kachroo A, Kachroo P (2014) Chemical inducers of systemic immunity in plants. Journal of Experimental Botany 65 (7):1849-1855. doi:10.1093/jxb/eru010 
Han M-H, Goud S, Song L, Fedoroff N (2004) The Arabidopsis double-stranded RNAbinding protein HYL1 plays a role in microRNA-mediated gene regulation.

Proceedings of the National Academy of Sciences of the United States of America 101 (4):1093-1098. doi:10.1073/pnas.0307969100

Jeong R-D, Chandra-Shekara AC, Barman SR, Navarre D, Klessig DF, Kachroo A, Kachroo P (2010) Cryptochrome 2 and phototropin 2 regulate resistance proteinmediated viral defense by negatively regulating an E3 ubiquitin ligase. Proceedings of the National Academy of Sciences, USA 107 (30):13538-13543. doi:10.1073/pnas.1004529107

Jia Y, McAdams SA, Bryan GT, Hershey HP, Valent B (2000) Direct interaction of resistance gene and avirulence gene products confers rice blast resistance. The EMBO Journal 19 (15):4004-4014. doi:10.1093/emboj/19.15.4004

Jones JDG, Dangl JL (2006) The plant immune system. Nature 444 (7117):323-329

Kachroo P (2006) Host gene-mediated virus resistance mechanisms and signaling in Arabidopsis. In: Loebenstein G, Carr JP (eds) Natural Resistance Mechanisms of Plants to Viruses. Springer Netherlands, pp 147-164. doi:10.1007/1-4020-3780$5 \_7$

Lau OS, Deng XW (2012) The photomorphogenic repressors COP1 and DET1: 20 years later. Trends in Plant Science 17 (10):584-593. doi:10.1016/j.tplants.2012.05.004

Lim G-H, Hoey T, Zhu S, Clavel M, Yu K, Navarre D, Kachroo A, Deragon J-M, Kachroo P (2018) COP1, a negative regulator of photomorphogenesis, positively regulates plant disease resistance via double-stranded RNA binding proteins. PLOS Pathogens 14 (3):e1006894. doi:10.1371/journal.ppat.1006894

Mackey D, Holt BF, Wiig A, Dangl JL (2002) RIN4 interacts with Pseudomonas syringae type III effector molecules and is required for RPM1-mediated resistance in Arabidopsis. Cell 108 (6):743-754

Mateo A, Mühlenbock P, Rustérucci C, Chang CC-C, Miszalski Z, Karpinska B, Parker JE, Mullineaux PM, Karpinski S (2004) LESION SIMULATING DISEASE 1 Is required for acclimation to conditions that promote excess excitation energy. Plant Physiology 136 (1):2818-2830. doi:10.1104/pp.104.043646

Raja P, Jackel JN, Li S, Heard IM, Bisaro DM (2014) Arabidopsis double-stranded RNA binding protein DRB3 participates in methylation-mediated defense against geminiviruses. J Virol 88. doi:10.1128/jvi.02305-13

Rustérucci C, Aviv DH, Holt BF, Dangl JL, Parker JE (2001) The disease resistance signaling components EDS1 and PAD4 are essential regulators of the cell death pathway controlled by LSD1 in Arabidopsis. The Plant Cell 13 (10):2211-2224. doi:10.1105/tpc.010085

Schulze-Lefert P (2004) Plant immunity: The origami of receptor activation. Current Biology 14 (1):R22-R24

Scofield SR, Tobias CM, Rathjen JP, Chang JH, Lavelle DT, Michelmore RW, Staskawicz BJ (1996) Molecular basis of gene-for-gene specificity in bacterial speck disease of tomato. Science 274 (5295):2063-2065. doi:10.1126/science.274.5295.2063

Spoel SH, Mou Z, Tada Y, Spivey NW, Genschik P, Dong X (2009) Proteasomemediated turnover of the transcription coactivator NPR1 plays dual roles in regulating plant immunity. Cell 137 (5):860-872 
Todd LR, Fumiaki K (2000) A resistance gene product of the nucleotide binding siteleucine rich repeats class can form a complex with bacterial avirulence proteins in vivo. The Plant Journal 22 (4):345-354. doi:doi:10.1046/j.1365-

313x.2000.00744.x

Tsutomu K, Jaesung N, C. BD, F. HB, A. HD, Aaron W, L. DJ (2005) A duplicated pair of Arabidopsis RING-finger E3 ligases contribute to the RPM1- and RPS2mediated hypersensitive response. The Plant Journal 44 (2):258-270. doi:doi:10.1111/j.1365-313X.2005.02525.X

Vaucheret H, Vazquez F, Crété P, Bartel DP (2004) The action of ARGONAUTE1 in the miRNA pathway and its regulation by the miRNA pathway are crucial for plant development. Genes \& Development 18 (10):1187-1197. doi:10.1101/gad.1201404

Vazquez F, Gasciolli V, Crété P, Vaucheret H (2004) The nuclear dsRNA binding protein HYL1 is required for microRNA accumulation and plant development, but not posttranscriptional transgene silencing. Current Biology 14 (4):346-351. doi:https://doi.org/10.1016/j.cub.2004.01.035

Venugopal SC, Jeong R-D, Mandal MK, Zhu S, Chandra-Shekara AC, Xia Y, Hersh M, Stromberg AJ, Navarre D, Kachroo A, Kachroo P (2009) Enhanced disease susceptibility 1 and salicylic acid act redundantly to regulate resistance genemediated signaling. PLOS Genetics 5 (7):e1000545. doi:10.1371/journal.pgen.1000545

Wu F, Yu L, Cao W, Mao Y, Liu Z, He Y (2007) The N-terminal double-stranded RNA binding domains of Arabidopsis HYPONASTIC LEAVES1 are sufficient for premicroRNA processing. The Plant Cell 19 (3):914-925. doi:10.1105/tpc. 106.048637

Xiaozhen H, Yansha L, Xiaoyan Z, Jianru Z, Shuhua Y (2010) The Arabidopsis LSD1 gene plays an important role in the regulation of low temperature-dependent cell death. New Phytologist 187 (2):301-312. doi:doi:10.1111/j.14698137.2010.03275.X

Yang SW, Chen HY, Yang J, Machida S, Chua NH, Yuan YA (2010) Structure of Arabidopsis HYPONASTIC LEAVES1 and its molecular implications for miRNA processing. Structure 18. doi:10.1016/j.str.2010.02.006

Zhu S, Jeong RD, Lim GH, Yu K, Wang C, Chandra-Shekara AC (2013) Doublestranded RNA-binding protein 4 is required for resistance signaling against viral and bacterial pathogens. Cell Reports 4. doi:10.1016/j.celrep.2013.08.018

Zuo Z-C, Meng Y-Y, Yu X-H, Zhang Z-L, Feng D-S, Sun S-F, Liu B, Lin C-T (2012) A study of the blue-light-dependent phosphorylation, degradation, and photobody formation of Arabidopsis CRY2. Molecular Plant 5 (3):726-733. doi:https://doi.org/10.1093/mp/sss007 


\section{Figure Legends}

Figure 1. DRB proteins are required for local resistance to Pseudomonas avrRpm1. (A) Western blots showing relative levels of DRB1-MYC, DRB2-MYC and DRB4-MYC in avrRPM1 $P$. syringae (Pst) inoculated $d r b$ plants expressing $D R B$ transgenes under their native promoters. Leaves were sampled at 0,24 and $48 \mathrm{~h}$ post inoculation. Ponceau-S staining of the Western blot was used as the loading control. Numbers below each panel indicate relative levels of Rubisco quantified using Image Quant software. This experiment was repeated two times with similar results. (B) Growth of avirulent avrRpm1 Pst strain on Col-0 and $d r b$ mutants. Error bars indicate SD. Asterisks indicate data statistically significant from that of control $(\mathrm{Col}-0)(\mathrm{P}<0.001, \mathrm{n}=4)$. NS indicates that the data was not significantly different from Col-0. These experiments were repeated five times with similar results. (C) Electrolyte leakage in Col-0 plants infiltrated with $\mathrm{MgCl}_{2}$ or avrRpm1 Pst. Error bars represent SD. This experiment was repeated two times with similar results. Asterisks indicate data significantly different from those of $\operatorname{Col}-0(\mathrm{P}<0.001, \mathrm{n}=6)$. (D) Levels of free SA in mock $\left(\mathrm{MgCl}_{2}\right)$ and pathogen (avrRpm1 Pst) inoculated Col-0 and $d r b$ plants $48 \mathrm{~h}$ post treatments. Error bars indicate SD. Asterisks indicate statistically higher levels of SA compared to respective mock-inoculated plants $(\mathrm{P}<0.0001, \mathrm{n}=4)$. "a" indicates significantly lower levels of SA in $d r b 1$ and $d r b 2$ plants compared to pathogen-inoculated Col-0 plants. (E) Quantitative RT-PCR analysis showing relative levels of $P R-1$ transcript in mock and avrRpm1 Pst inoculated Col-0 and $d r b$ plants. This experiment was repeated twice using two or more independent cDNA preparations as templates. Asterisks indicate statistically higher expression compared to respective mock-inoculated plants $(\mathrm{P}<0.0001, \mathrm{n}=4)$. "a" indicates significantly lower expression compared to pathogen-inoculated Col-0 plants.

Figure 2. C-terminal truncated DRB1 protein does not complement defense phenotypes of $\boldsymbol{d r b 1}$ plants. (A and B) Growth of avrRpm1 Pst (A) and virulent (B) DC3000 strains on Col-0, $d r b 1$, and $d r b 1$ expressing wild-type (drb1::DRB1) or Cterminal truncated DRB1 ( $\left.d r b 1:: \Delta^{c} D R B 1\right)$. Error bars indicate SD. Asterisks indicate data significantly different from those of Col-0 $(\mathrm{P}<0.0001, \mathrm{n}=4)$. These experiments were repeated two times with similar results. (C) Quantitative RT-PCR analysis showing relative levels of $P R-1$ transcript in mock and avrRpm1 Pst inoculated Col-0, $d r b 1$, and $d r b 1$ expressing wild-type (drb1::DRB1) or C-terminal truncated DRB1 (drb1:: $\triangle^{c} D R B 1$ ). This experiment was repeated twice using two or more independent cDNA preparations as templates. Asterisks indicate data significantly higher compared to respective mock-inoculated plants $(P<0.0001, n=4)$. "a" 
indicates significantly lower expression compared to pathogen-inoculated Col-0 plants. (D) Growth of avirulent avrRpm1 Pst strain on Col-0, ago1-27, and dcl1-7 plants. These experiments were repeated two times with similar results.

Figure 3. DRB1 and DRB4 proteins are required for the stability of RPM1. (A) Western blots showing relative levels of RPM1-MYC in $d r b$ mutants. Ponceau-S staining of the Western blot was used as the loading control. Arrow indicates RPM1MYC protein. This experiment was repeated three times with similar results. (B) Growth of avrRpm1 Pst on Col-0, drb1, drb4 and rpm1 plants. Error bars indicate SD. Asterisks indicates significantly higher growth compared to respective mockinoculated plants $(\mathrm{P}<0.0007, \mathrm{n}=4)$. " $\mathrm{b}$ " indicates significant difference between rpm1 and $d r b$ plants $(\mathrm{P}<0.003, \mathrm{n}=4)$. (C) Growth of virulent Pst (DC3000) on Col-0, $d r b$ and sid2 plants that were pretreated with water or SA $(500 \mu \mathrm{m})$ prior to inoculation. Error bars indicate SD. Asterisks indicate data statistically significant from that of water treated plants $(\mathrm{P}<0.002, \mathrm{n}=4)$. (D) SAR response in Col-0 and $d r b$ plants. Primary leaves were inoculated with $\mathrm{MgCl}_{2}$ or $P$. syringae expressing avrRpm1. The distal leaves were inoculated with the virulent $P$. syringae and growth of the virulent bacteria was monitored at 3 dpi. Asterisks indicates statistically lower growth compared to respective $\mathrm{MgCl}_{2}$ inoculated plants $(\mathrm{P}<0.001, \mathrm{n}=4)$. This experiment was repeated three times with similar results.

Figure 4. Pathogen-triggered degradation of RPM1 is not required for its downstream signaling. (A) Western blot showing RPM1-MYC levels in Col0::RPM1-MYC plants at 0-3 hours after avrRpm1 inoculation. The plants were treated with DMSO solvent, protease inhibitor cocktail (PI), or MG132 and inoculated with $10^{6}$ bacteria $24 \mathrm{~h}$ after treatment. Ponceau-S staining of the Western blot was used as the loading control. This experiment was repeated two times with similar results. (B) Electrolyte leakage in Col-0 plants infiltrated with $\mathrm{MgCl}_{2}$ or avrRpm1 Pst. Error bars represent SD (n=6). This experiment was repeated two times with similar results. (C) RNA gel blot showing time-course expression analysis of $P R-1$ in plants treated with DMSO, PI or MG132 $24 \mathrm{~h}$ prior to inoculation with avrRpm1 Pst. Total RNA was extracted from inoculated leaves at 24 hours post inoculation (hpi). Ethidium bromide staining of rRNA was used as the loading control. The experiment was repeated twice with similar results. (D) Growth of avrRpm1 Pst on Col-0 plants treated with DMSO, PI or MG132 $24 \mathrm{~h}$ prior to inoculation with avrRpm1 Pst. Error bars indicate SD. (E) Western blot showing RPM1-MYC levels in $L S D 1$ and $l s d 1$ plants. Both genotypes were derived from a cross between Col-0::RPM1-MYC x lsd1 (Ws-0) plants and were homozygous for the rpm1 locus. Ponceau-S staining of the Western blot was used as the loading control. Arrow 
indicates RPM1-MYC protein. This experiment was repeated two times with similar results. (F) Western blot showing RPM1-MYC levels in LSD1::RPM1-MYC or Isd1::RPM1-MYC plants at 0, 3 or 6 hours after avrRpm1 inoculation. The RPM-MYC genotypes were homozygous for the rpm1 locus. Arrow indicates RPM1-MYC protein. This experiment was repeated two times with similar results. (G) Quantitative RT-PCR analysis showing relative levels of RPM1 transcript in LSD1 and lsd1 plants. Error bars indicate SD. This experiment was repeated twice using two or more independent cDNA preparations as templates. Asterisks indicate statistically higher expression compared to LSD1 plants $(\mathrm{P}<0.0001, \mathrm{n}=4)$.

Figure 5. DRB3 and DRB5 negatively regulate resistance to bacterial pathogens. (A) Typical morphological phenotypes of soil grown four-week-old $d r b$ single and double mutants. Scale bar, $0.5 \mathrm{~cm}$. (B and C) Growth of virulent DC3000 (B) and avirulent avrRpm1 Pst (C) P. syringae (Pst) strains on Col-0 and drb mutants. Error bars indicate SD. Asterisks indicate significantly higher growth compared to Col-0 $(\mathrm{P}<0.006, \mathrm{n}=4)$. NS indicates that the data were not significantly different from Col-0. " $b$ " indicates significant difference among indicated genotypes $(\mathrm{P}<0.003$, $\mathrm{n}=4$ ). (D) Simplified model showing genetic interaction between DRB1, DRB2, DRB4 and DRB3 and DRB5. A mutation in DRB1, DRB2 and DRB4 compromises both basal and R-mediated resistance against Pseudomonas syringae (Pst). In contrast, a mutation in DRB3 and DRB5 has no effect on host resistance to Pst but does suppress susceptibility caused by mutation in DRB1, DRB2 or DRB4 in a partial (dashed line) or complete (solid line) manner.

Figure 6. Overexpression of DRB5 compromises RPM1-mediated resistance. (A) Typical morphological phenotypes of soil grown four-week-old Col-0 and transgenic plants overexpressing DRB3 or DRB5 transgenes in Col-0 background. Scale bar, $0.5 \mathrm{~cm}$. (B) Quantitative RT-PCR analysis showing relative levels of $D R B$ transcripts in transgenic Col-0 plants overexpressing DRB3 or DRB5 transgenes. This experiment was repeated twice using two independent cDNA preparations as templates. Error bars indicate SD. Asterisks indicate data significantly different from those of Col-0 ( $\mathrm{P}<0.0001, \mathrm{n}=4)$. (C) Growth of avrRpm1 Pst strain on Col-0 and DRB overexpressing plants. Error bars indicate SD. Asterisks indicate data significantly different from those of control $(\mathrm{Col}-0)(\mathrm{P}<0.008, \mathrm{n}=4)$. This experiment was repeated four times and while 35S-DRB5 plants showed enhanced susceptibility in all experiments, the 35S-DRB3 plants showed increased susceptibility in two of four experiments. (D) Electrolyte leakage in Col-0 or 35S-DRB plants infiltrated with $\mathrm{MgCl}_{2}$ or avrRpm1 Pst. Error bars represent SD (n=6). This experiment was repeated two times with similar results. 


\section{Supplementary Figure Legends}

S. Figure 1. DRB proteins are required for local resistance to Pseudomonas. (A) Typical morphological phenotypes of avrRpm1 inoculated leaves. The inoculated leaves were marked with silver ink and photographed $24 \mathrm{~h}$ post inoculation. (B and C) Growth of avirulent avrRpt2 (B) or virulent (C) Pst (DC3000) on Col-0 and $d r b$ mutants. Error bars indicate SD. Asterisks indicate data significantly different from those of Col-0 $(\mathrm{P}<0.001, \mathrm{n}=4)$. (D) RNA gel blot analysis showing expression of $P R-1$ in mock- and avrRpm1-inoculated Col-0 and $d r b$ plants. Total RNA was extracted from inoculated leaves at $24 \mathrm{hpi}$. Ethidium bromide staining of rRNA was used as the loading control. The experiment was repeated twice with similar results.

S. Figure 2. drb mutants show normal SA responsiveness. (A) Quantitative RTPCR analysis showing relative levels of RPM1-MYC transcript in Col-0 and $d r b$ mutants. This experiment was repeated twice using two independent cDNA preparations as templates. Error bars indicate SD. (B) Quantitative RT-PCR analysis showing relative levels of $P R-1$ transcript in Col-0 plants treated with SA, avrRpm1 or SA+avrRpm1. The leaves were sampled $24 \mathrm{~h}$ post treatment. This experiment was repeated twice using two or more independent cDNA preparations as templates. Asterisks indicate significantly higher expression compared to $\mathrm{MgCl}_{2}$ (mock) infiltrated plants $(\mathrm{P}<0.0001, \mathrm{n}=4)$. (C) RNA gel blot analysis showing expression of $P R-1$ in water- and SA-treated Col- 0 and $d r b$ plants. Total RNA was extracted from treated leaves at $24 \mathrm{hpi}$. Ethidium bromide staining of rRNA was used as the loading control. The experiment was repeated twice with similar results.

S. Figure 3. The Isd1 plants accumulate higher levels of basal and pathogeninduced reactive oxygen species. (A) $\mathrm{H}_{2} \mathrm{O}_{2}$ levels in LSD1::RPM1-MYC and Isd1::RPM1-MYC plants after mock $\left(\mathrm{MgCl}_{2}\right)$ or pathogen (avrRpm1) inoculation. The leaves were sampled $24 \mathrm{~h}$ post treatments and stained with DAB (3, 3diaminobenzidine). The experiment was repeated two times with similar results. (B) Typical morphological and microscopic cell death phenotypes shown by pathogen inoculated LSD1::RPM1-MYC and Isd1::RPM1-MYC plants. Leaves were photographed or stained $24 \mathrm{~h}$ post inoculation and at least six independent leaves were analyzed with similar results. (C) Growth of avirulent avrRpm1 Pst on LSD1::RPM1-MYC and lsd1::RPM1-MYC plants. The experiment was repeated twice with similar results. (D) Growth of avrRpm1 Pst on Col-0 and drb1 drb4 plants treated with DMSO or MG132 $24 \mathrm{~h}$ prior to inoculation. Error bars indicate SD. 
Asterisks indicate significantly higher growth compared to Col-0 plants $(\mathrm{P}<0.0001$, $\mathrm{n}=4$ ).

S. Figure 4. The drb3 drb5 double mutants show enhanced resistance to virulent bacteria (A and B) Growth of virulent (A) or avirulent avrRpm1 Pst (B) on Col-0, $d r b 3, d r b 5$ and $d r b 3 d r b 5$ double mutants. Error bars indicate SD. Asterisks indicate data significantly different from those of Col-0 $(\mathrm{P}<0.01, \mathrm{n}=4)$. The experiment was repeated twice with similar results.

S. Figure 5. Overexpression of DRB5 compromises basal and R-mediated resistance against bacterial pathogens. (A and B) Growth of avirulent avrRpt2 (A) or virulent DC3000 (B) Pst on Col-0 and plants overexpressing DRB3 or DRB5. Error bars indicate SD. Asterisks indicate data significantly different from those of Col-0 $(\mathrm{P}<0.01, \mathrm{n}=4)$. These experiments were repeated four times and while 355 DRB5 plants showed enhanced susceptibility in all experiments, the 35S-DRB3 plants showed increased susceptibility in two of four experiments. 
A

\begin{tabular}{lll}
\multicolumn{3}{c}{ avrRpm1 } \\
\hline 0 & $24 \quad 72$
\end{tabular}

$75-$

50 -

50

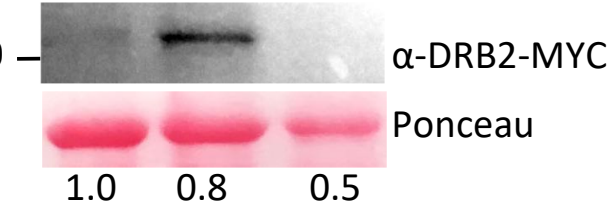

37

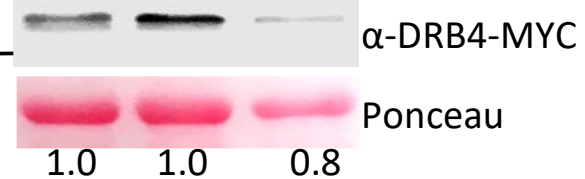

Figure 1

B

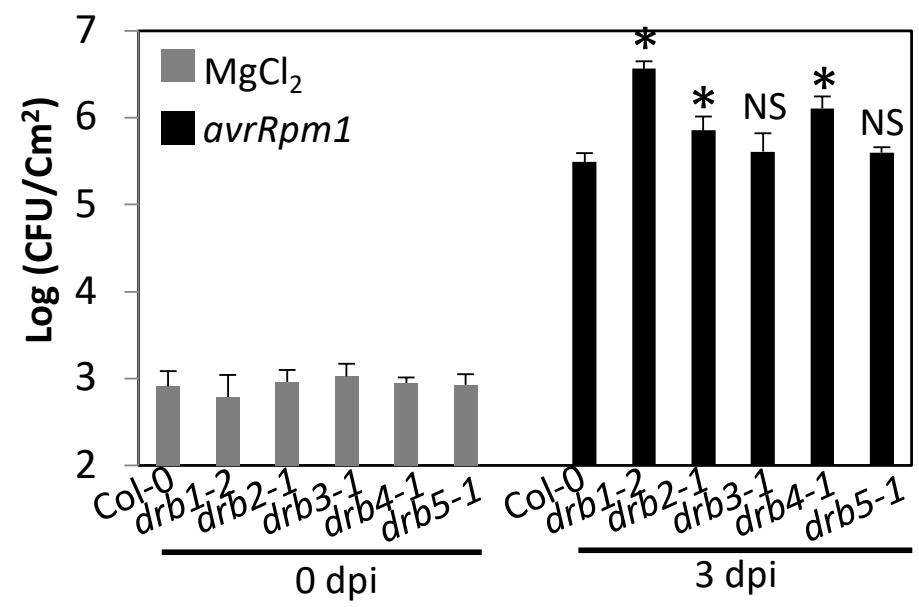

C

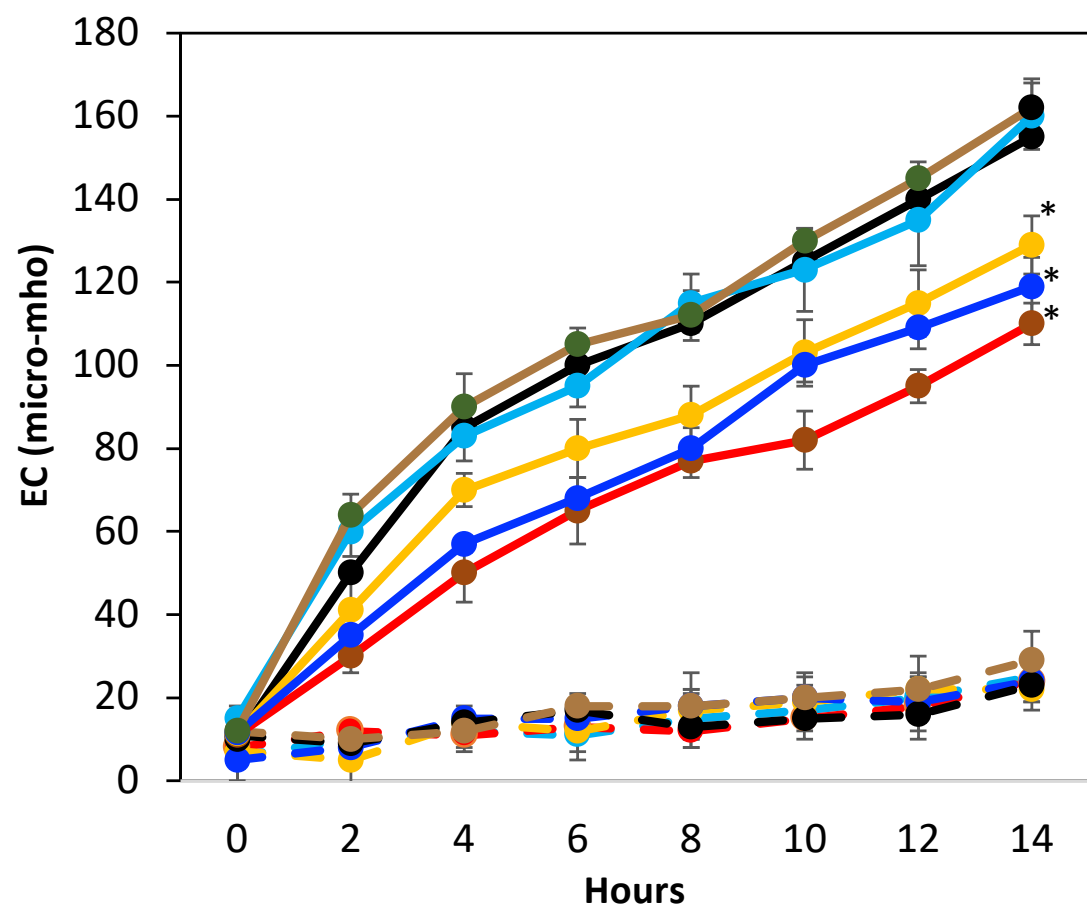

- Col-0- $\mathrm{MgCl}_{2}$

- - drb1- $\mathrm{MgCl}_{2}$

$=d b r 2-\mathrm{MgCl}_{2}$

$=d r b 3-\mathrm{MgCl}_{2}$

- $d r b 4-\mathrm{MgCl}_{2}$

$=d r b 5-\mathrm{MgCl}_{2}$

- Col-0- avr

- drb1- avr drb2-avr drb3- $a v r$ drb4- $a v r$ drb5- $a v r$

D

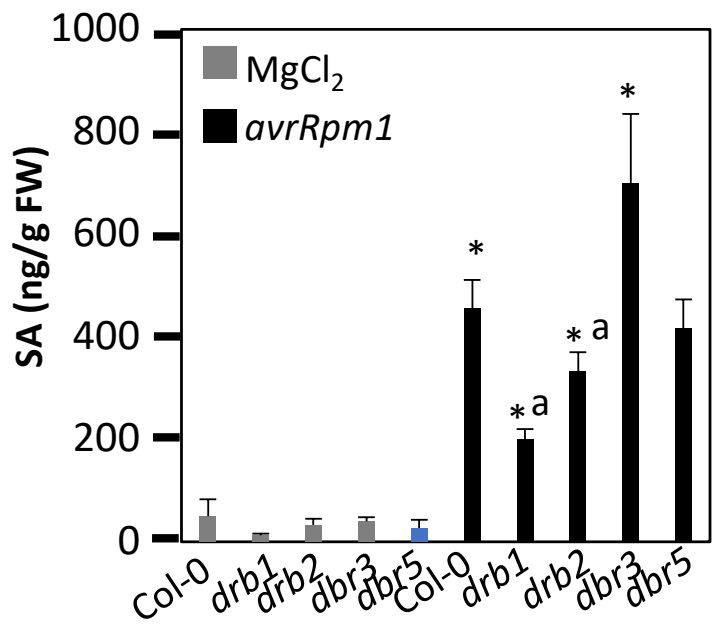

E

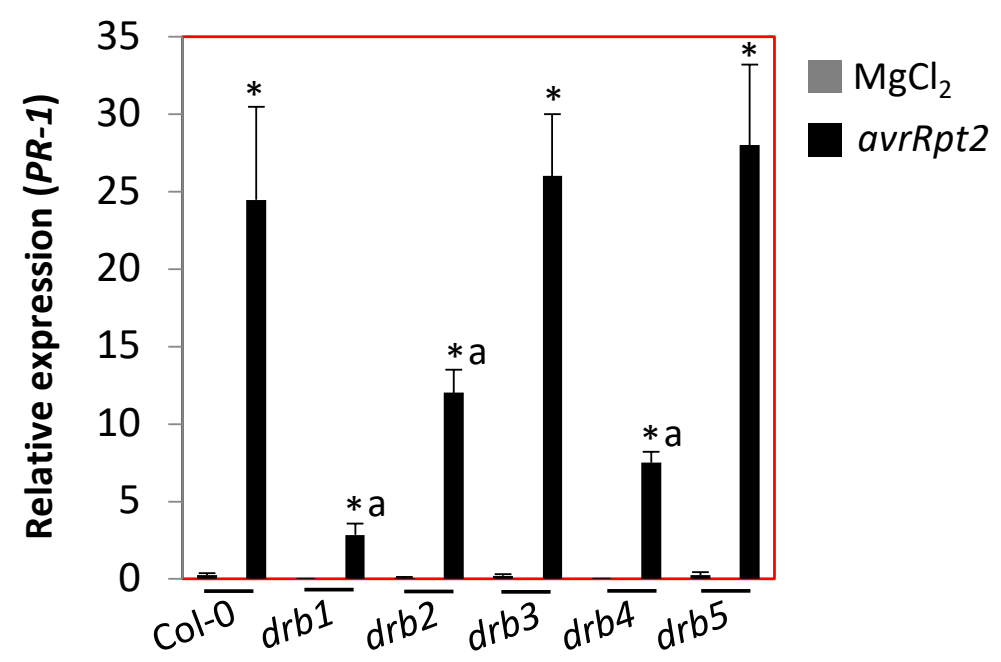


Figure 2
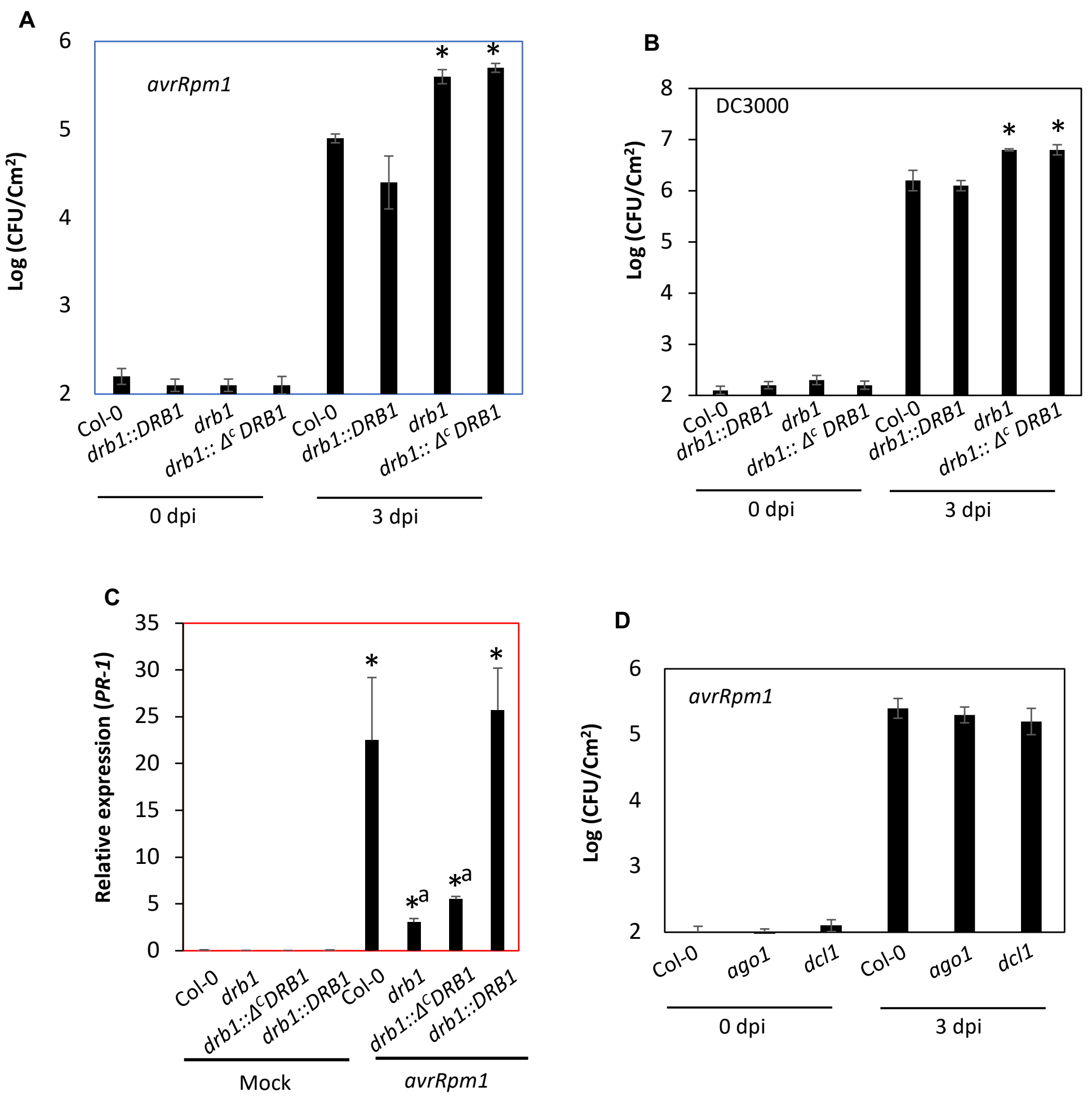
Figure 3
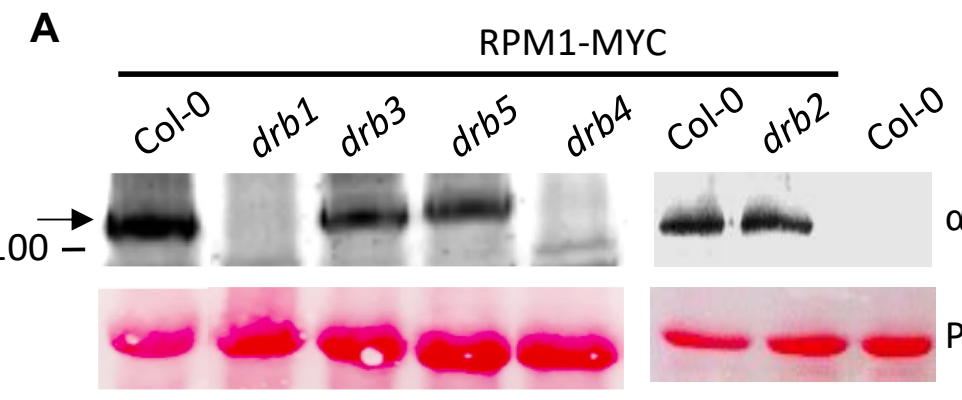

a-Mус

Ponceau

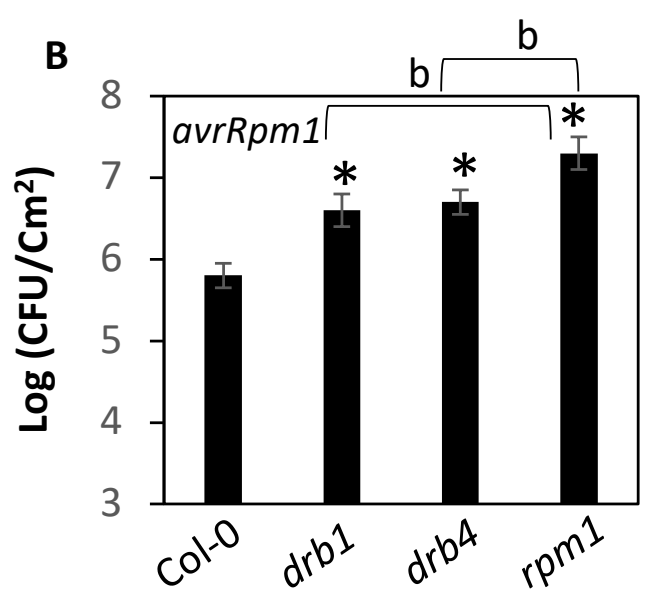

C

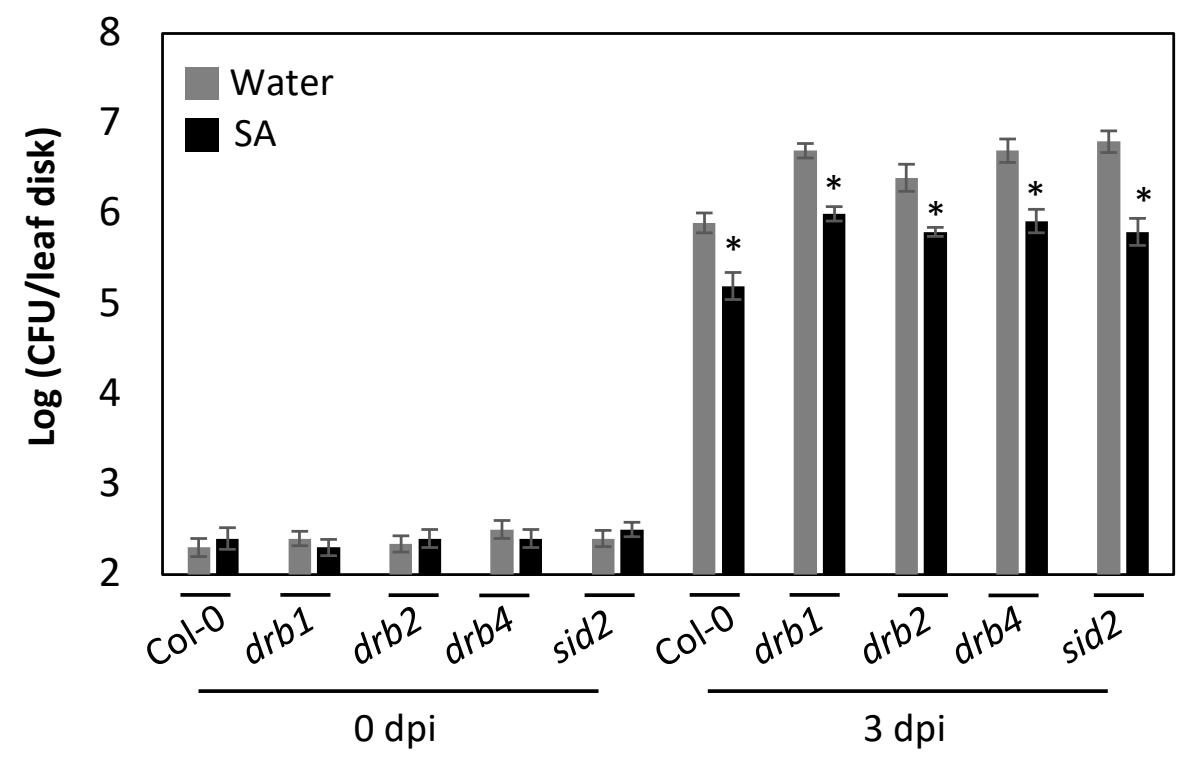

D

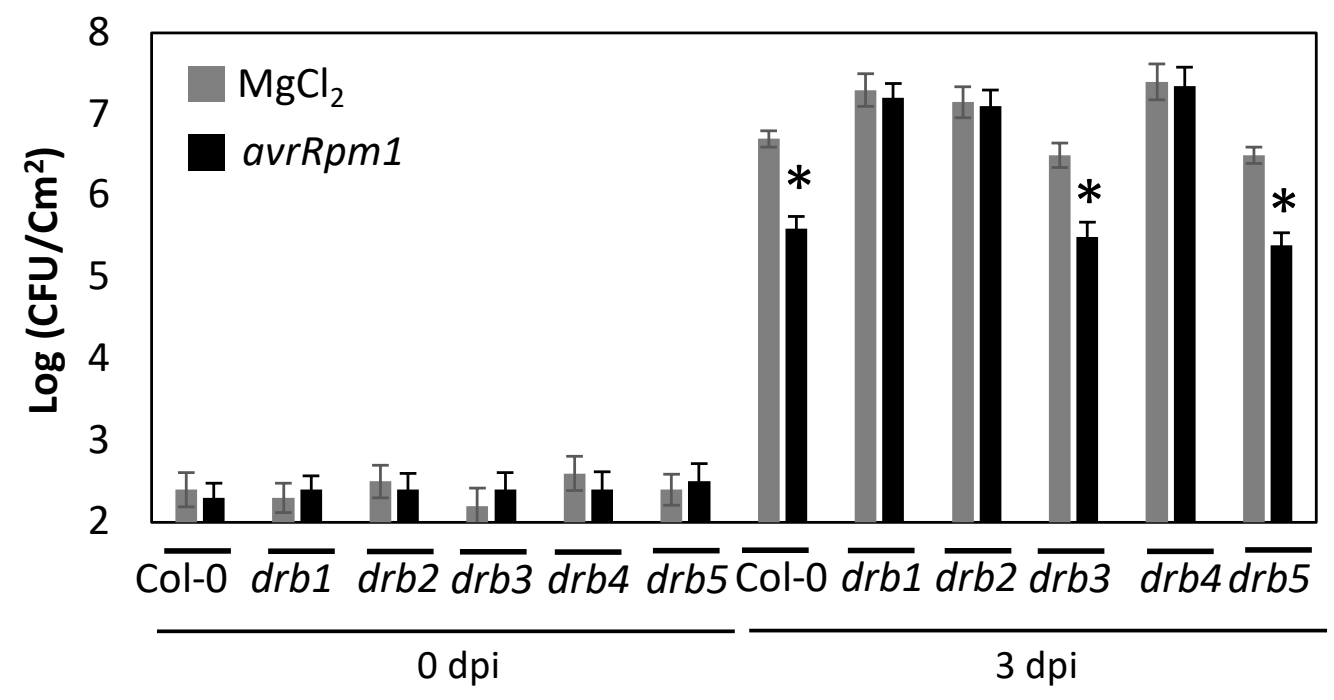




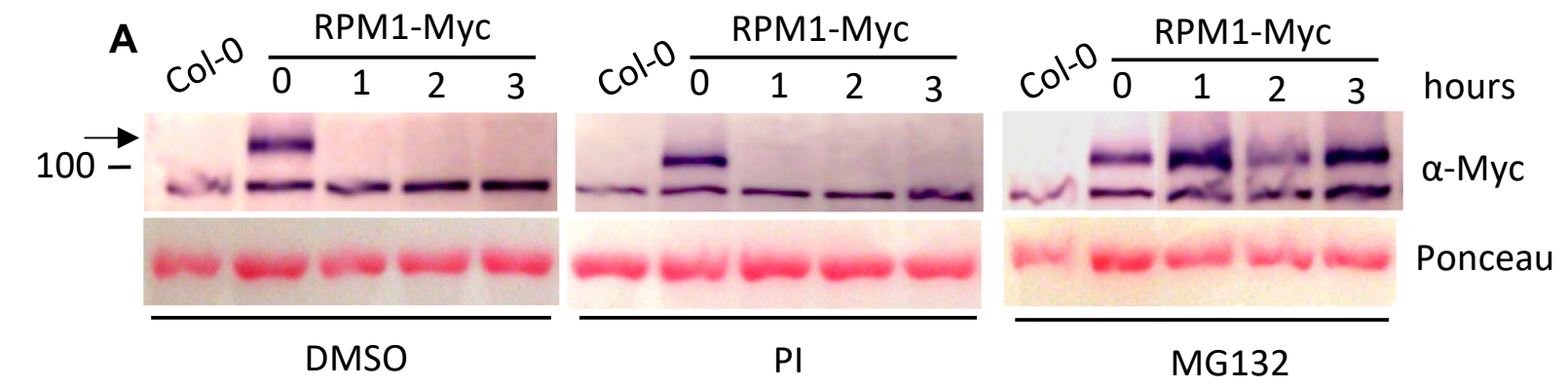

Figure 4

B

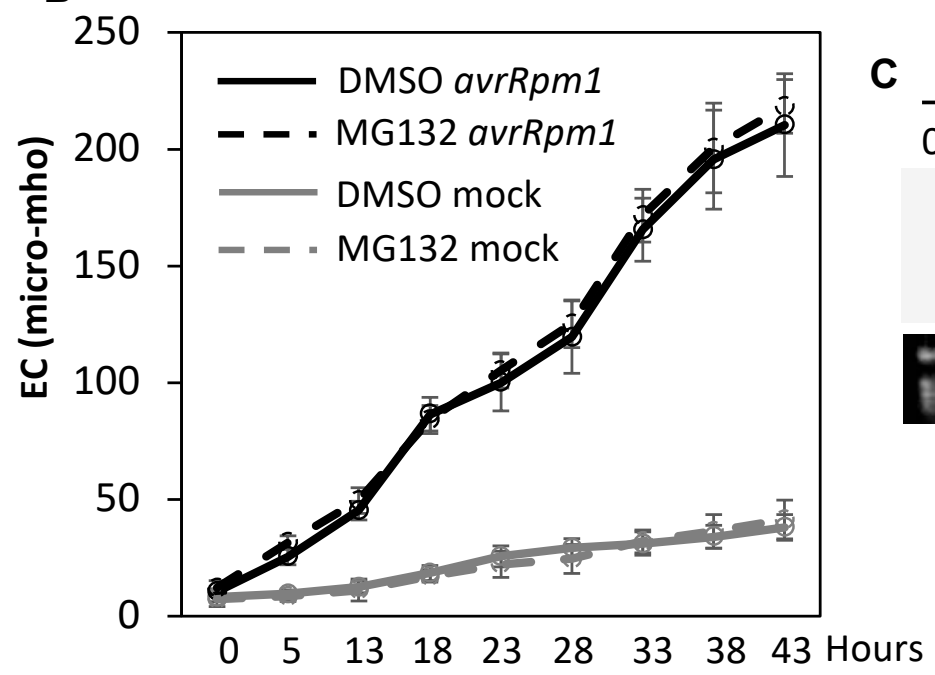

D

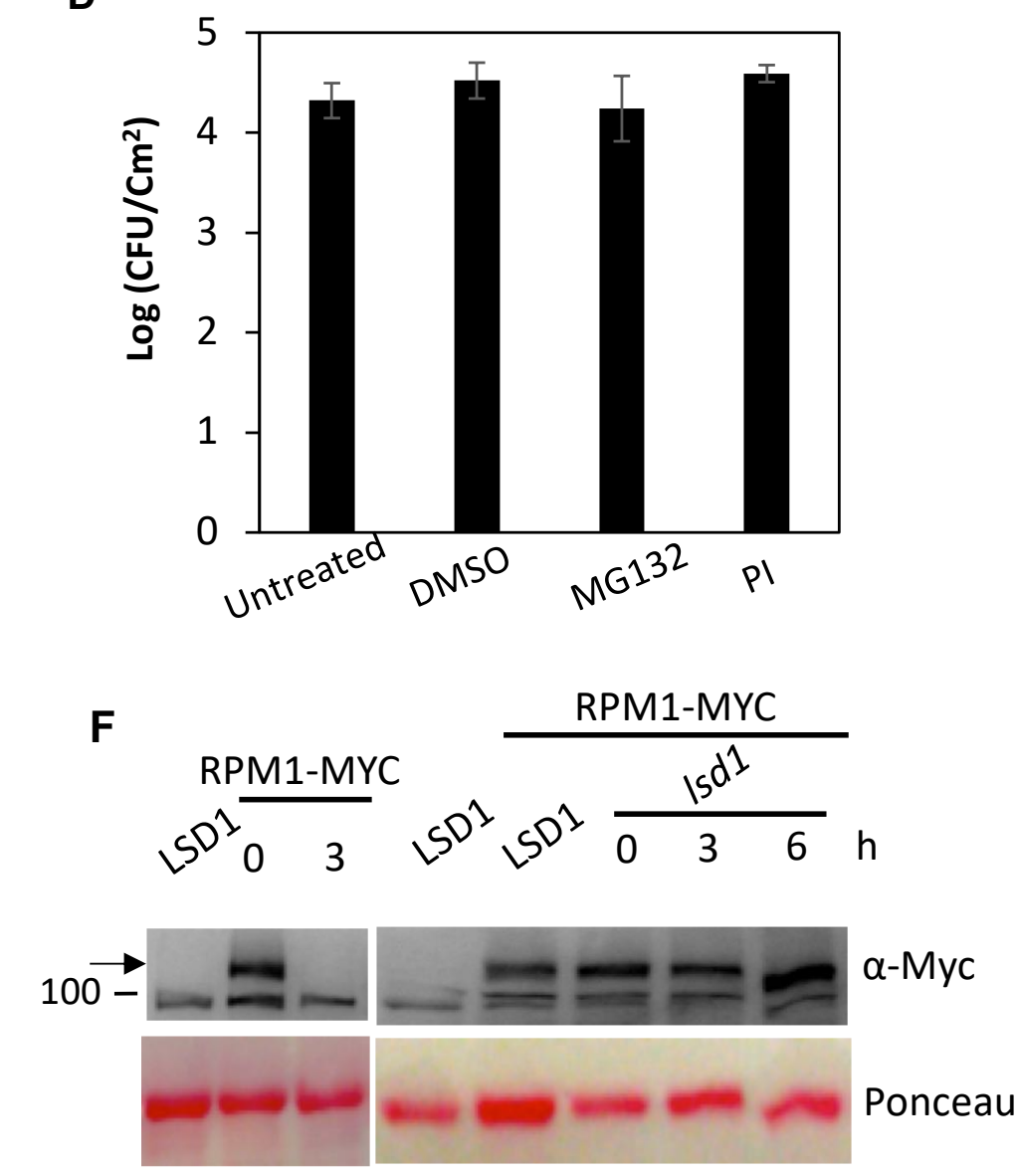

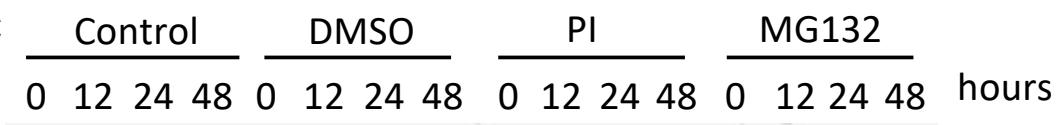

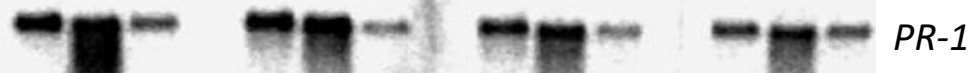

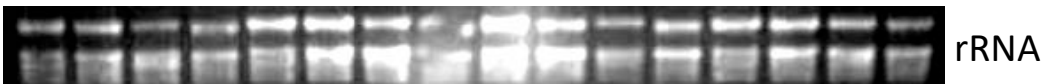

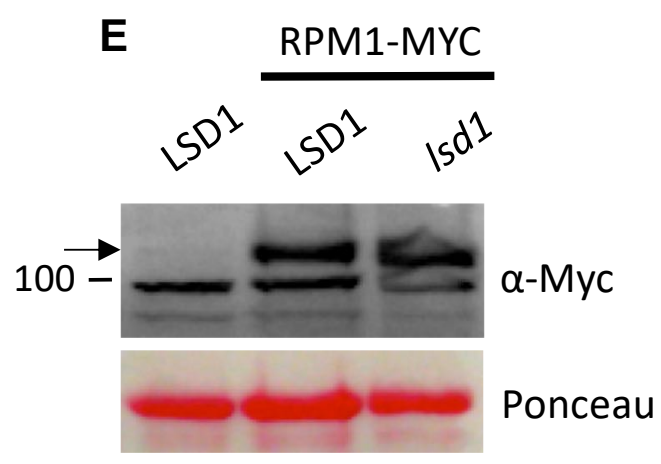

G

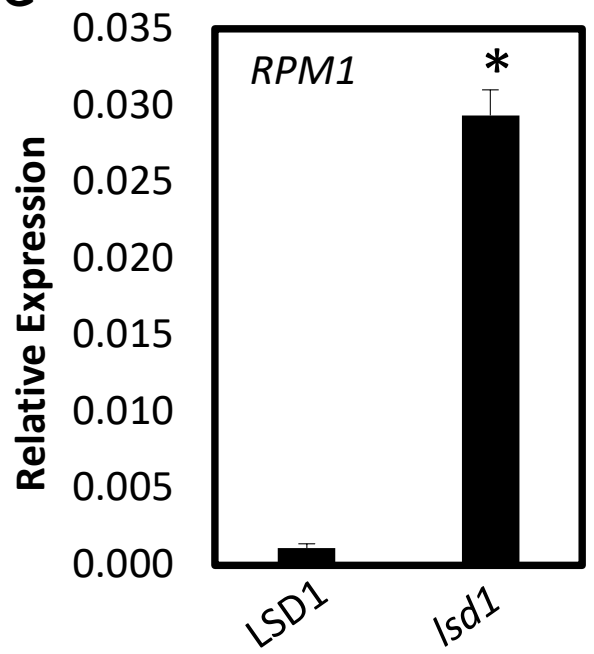


A

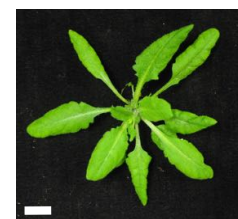

Col-0

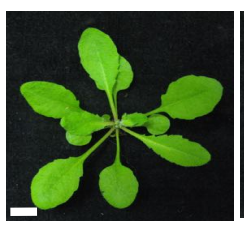

$d r b 2 d r b 3$

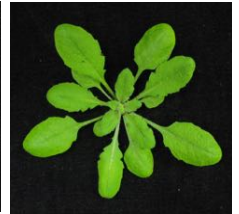

$d r b 2$

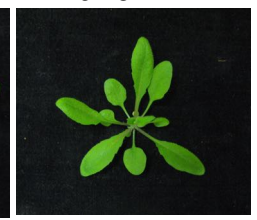

$d r b 2 d r b 4$

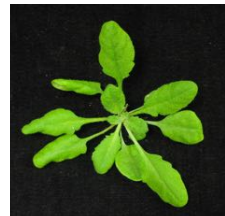

drb3

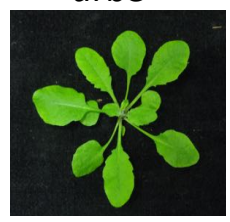

$d r b 2 d r b 5$

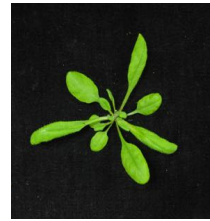

drb4

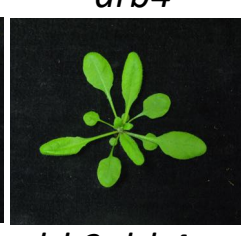

$d r b 3 d r b 4$

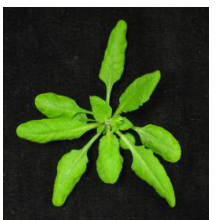

Figure 5

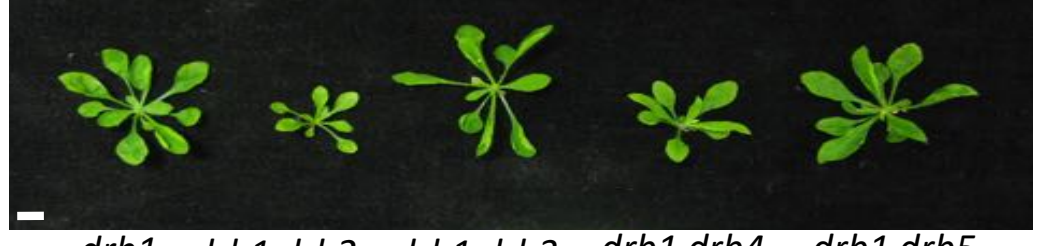

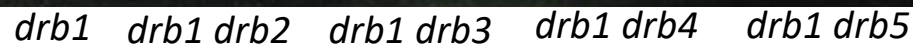

B

DC3000
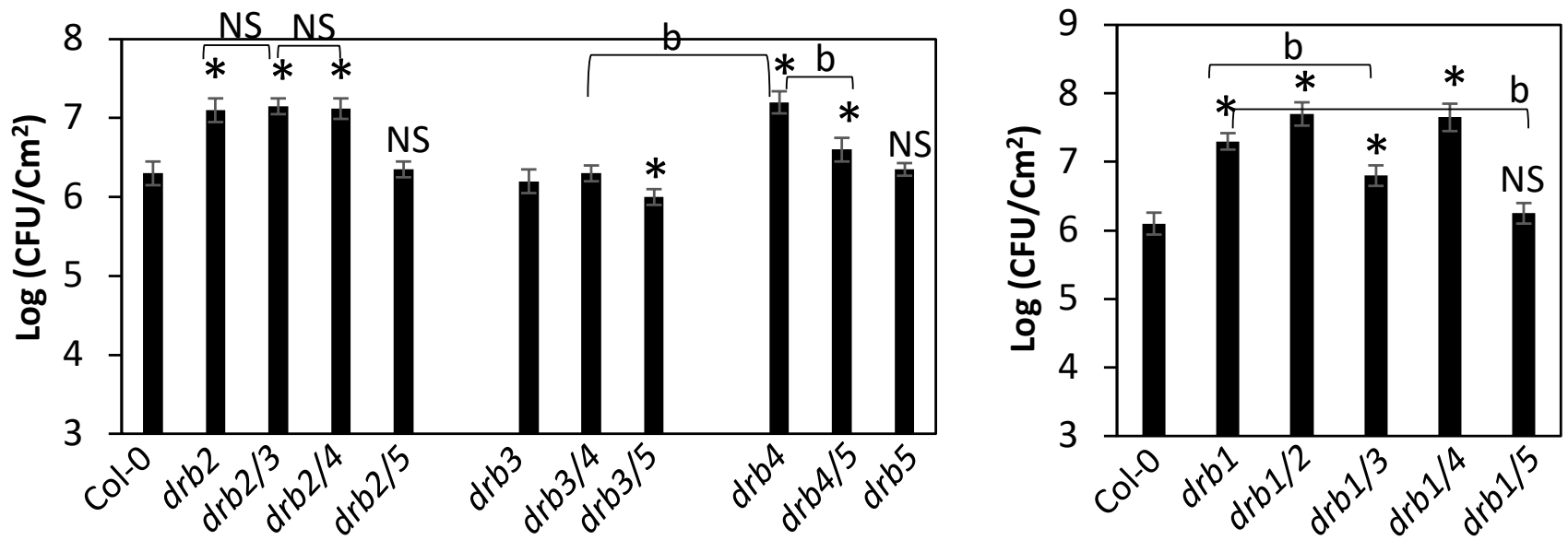

C
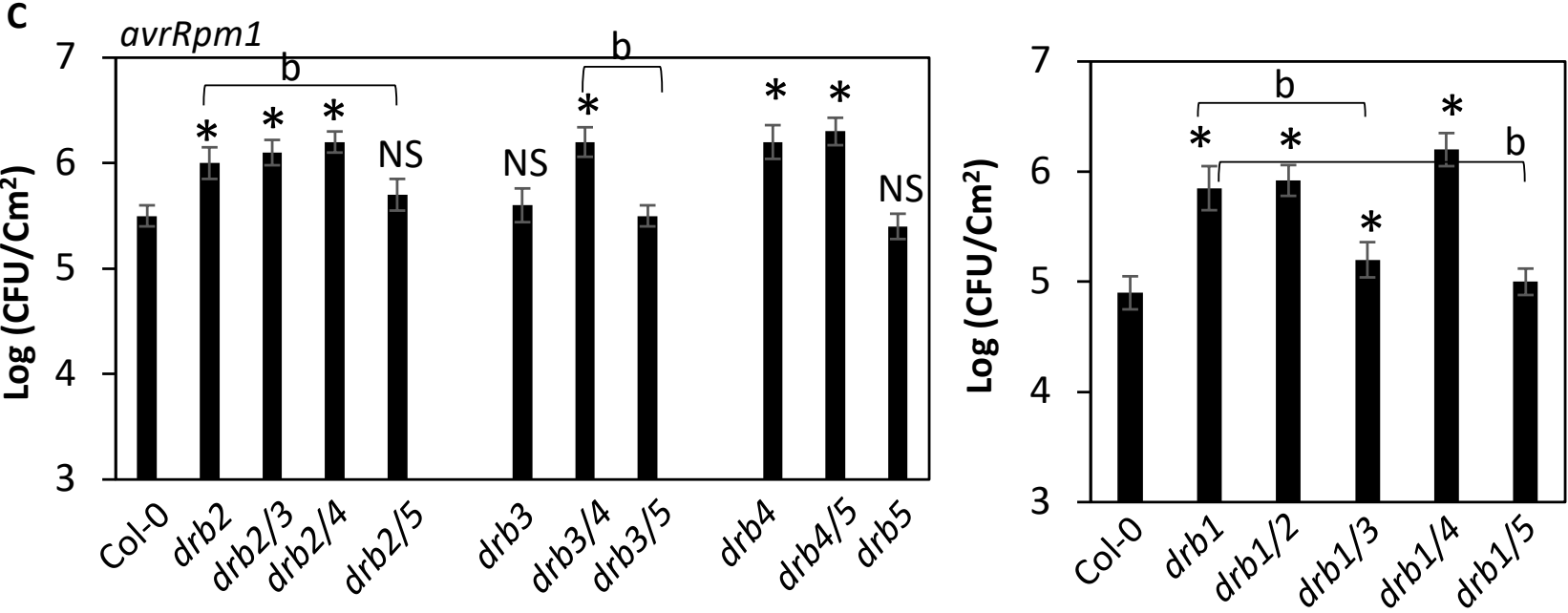

D

R-mediated Resistance

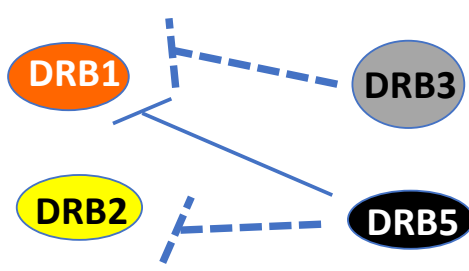

DRB4
Basal Resistance

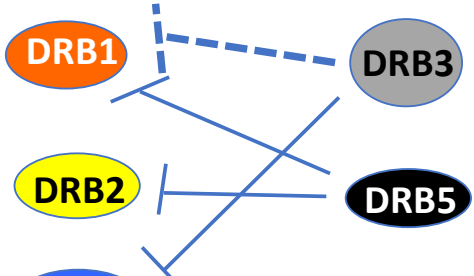


Figure 6

A

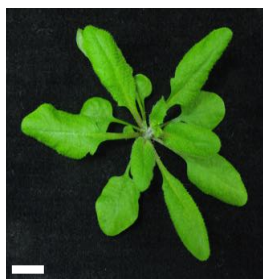

Col-0

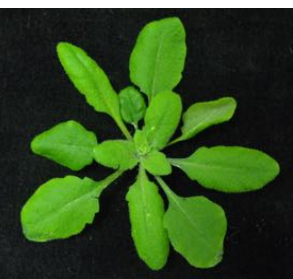

35S-DRB3

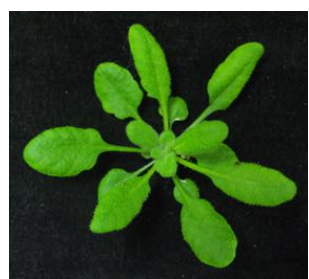

35S-DRB5
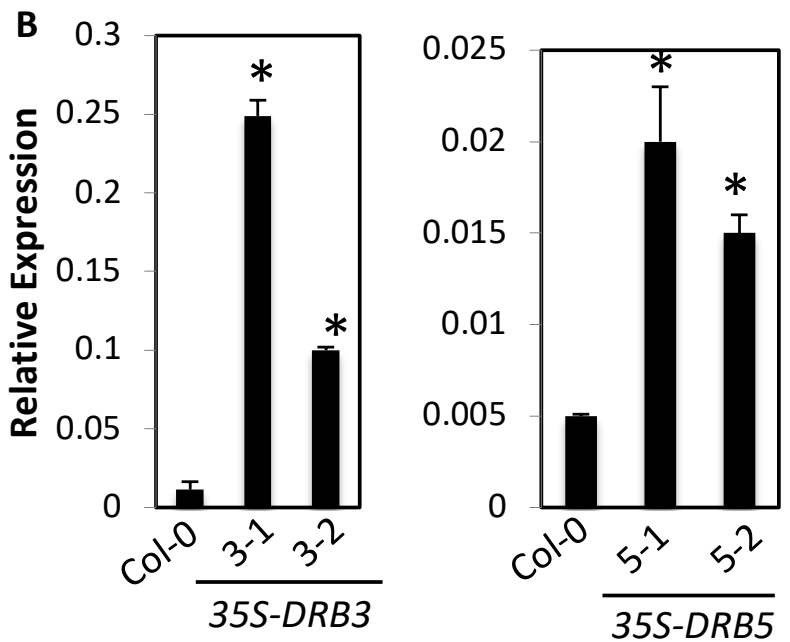

C

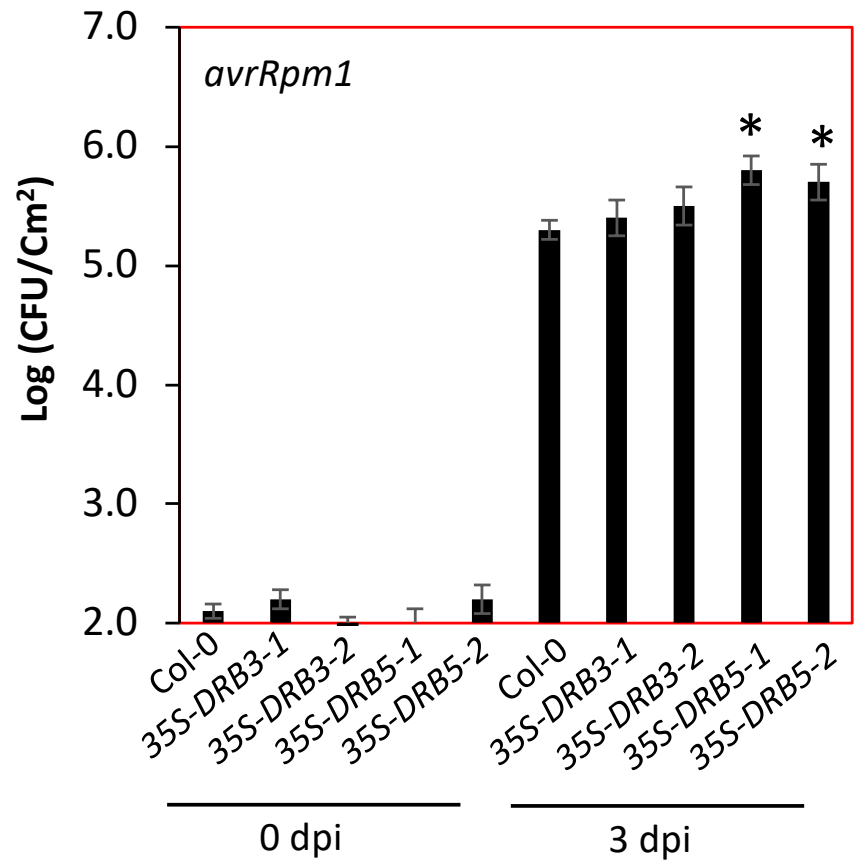

D

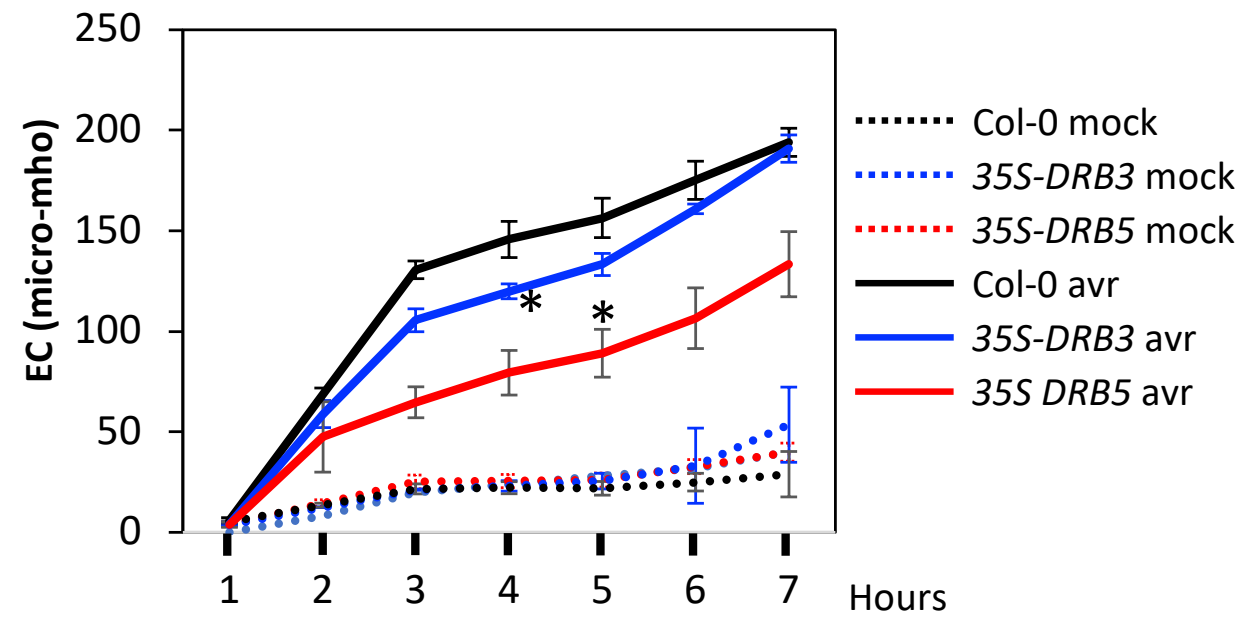


A

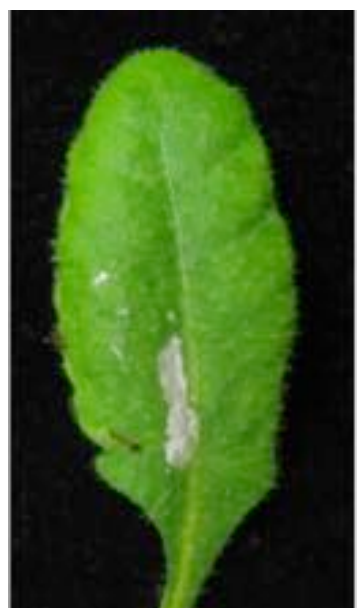

Col-0

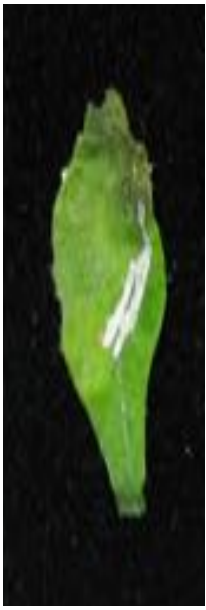

$d r b 1$

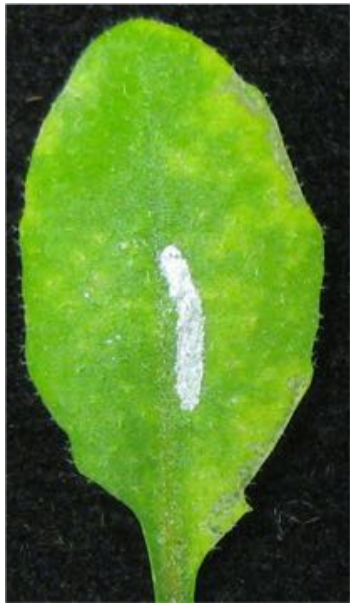

$d r b 2$

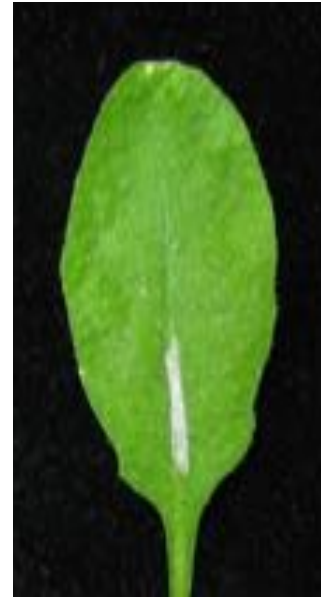

drb3

\section{S. Figure 1}

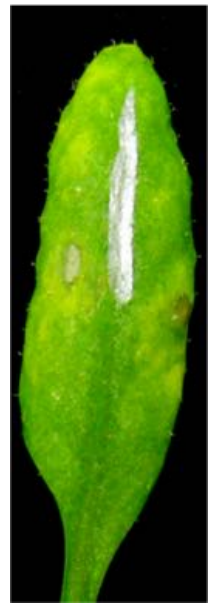

drb4

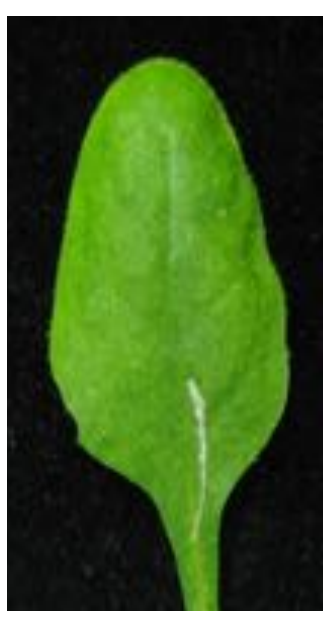

$d r b 5$

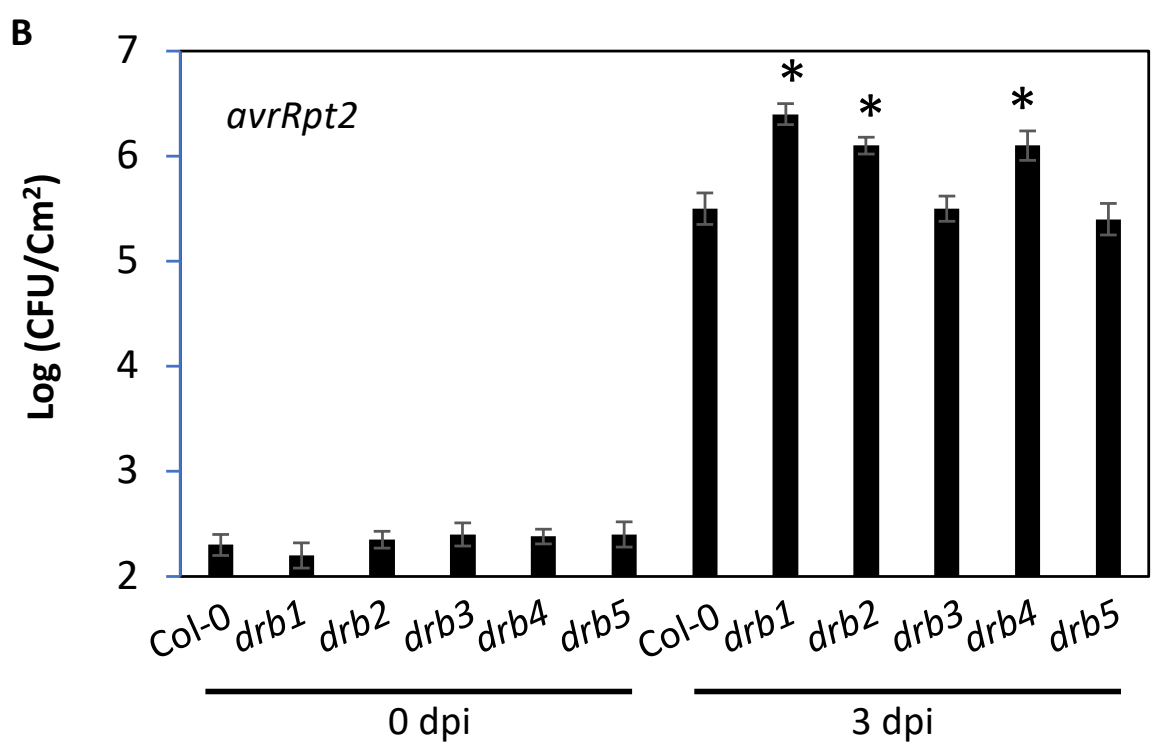

C

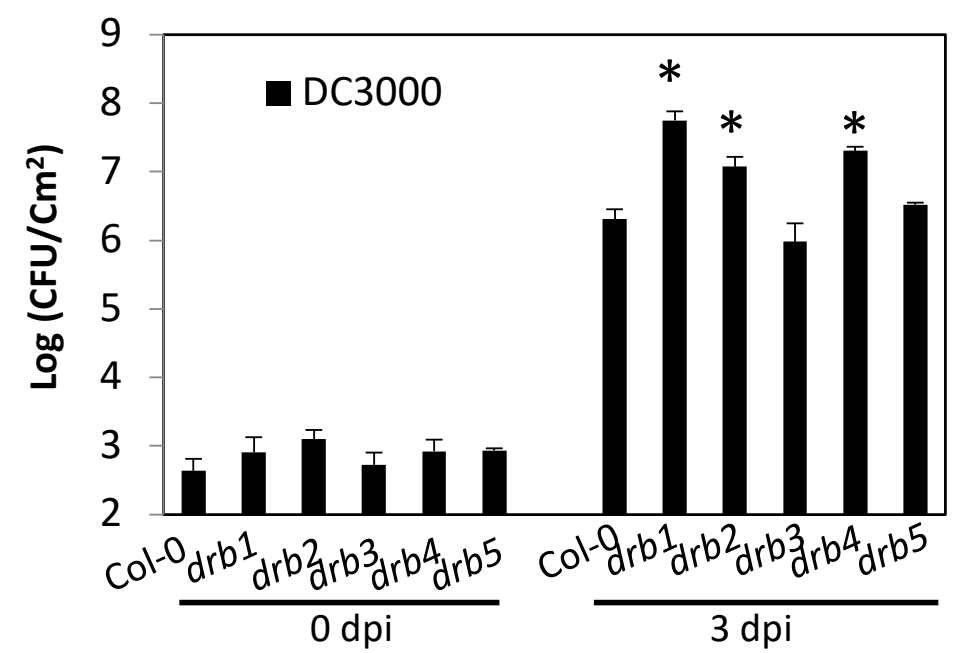

D

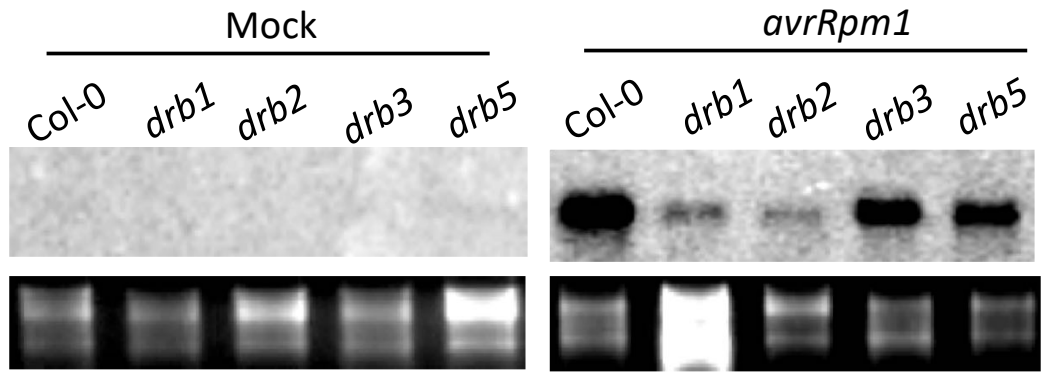

$P R-1$ 
A

S. Figure 2
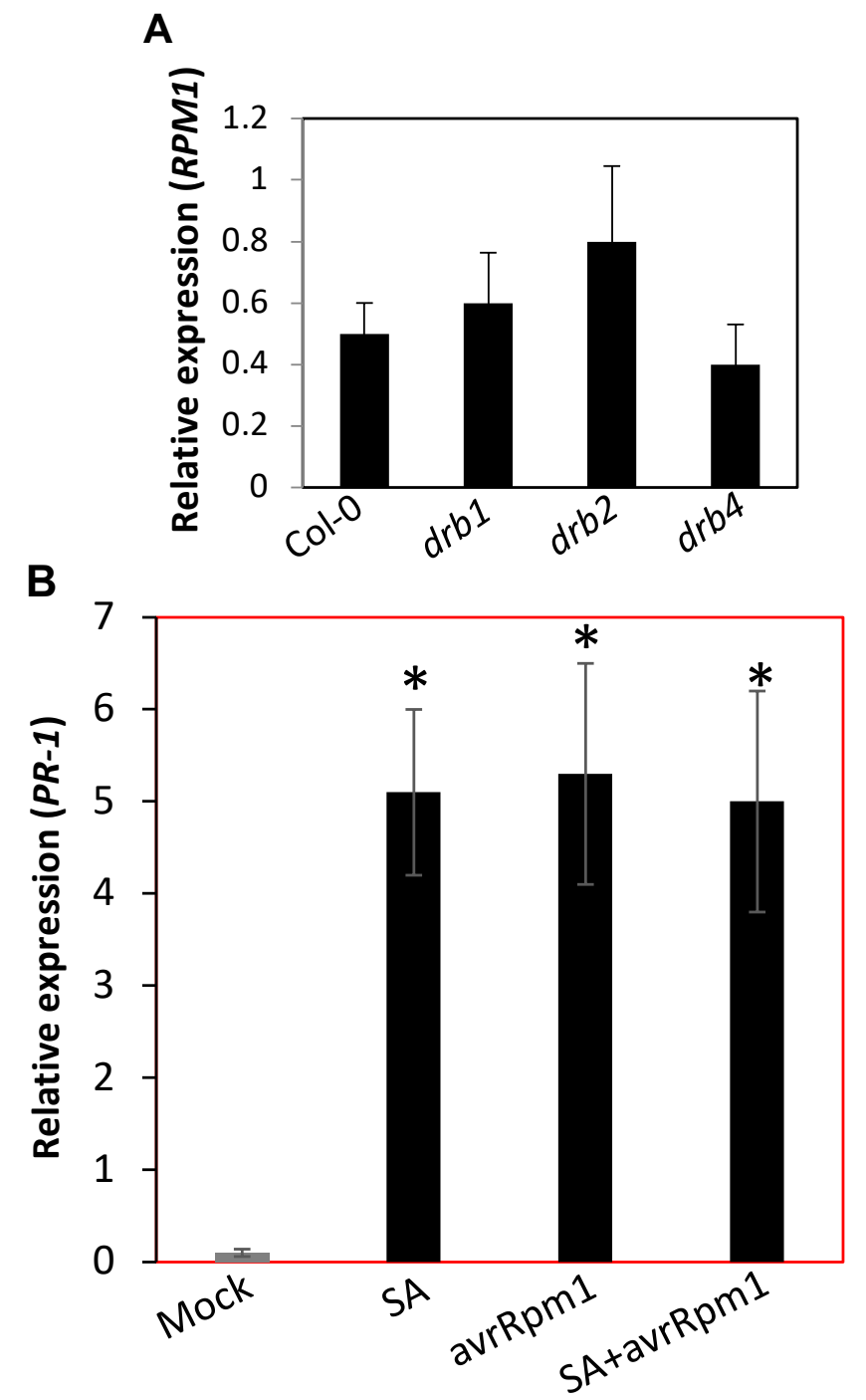

C

$\frac{\text { Water }}{c^{1-0} d r b^{1} d r b^{2} d r b^{3} d r b^{4} d r b^{5}} \frac{S A}{c^{1-0} d r b^{1} d r b^{2} d r b^{3} d r b^{4} d r b^{5}}$

PR1

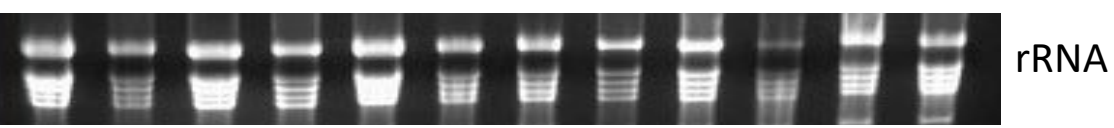




\section{S. Figure 3}

A

LSD1::RPM1-MYC Isd1::RPM1-MYC

Mock

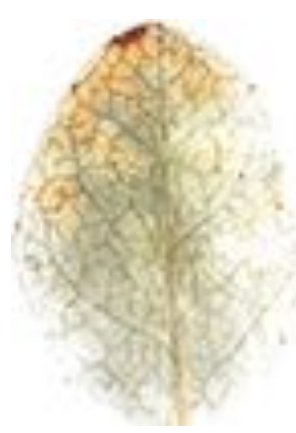

Avr
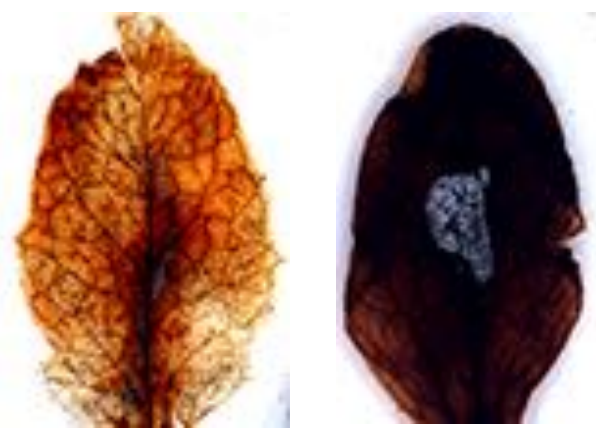

B

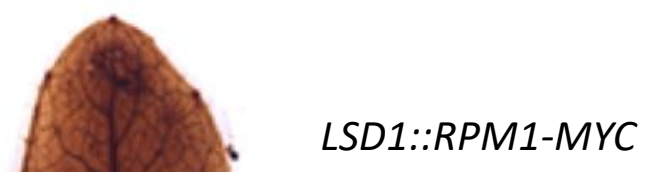

Isd1::RPM1-MYC
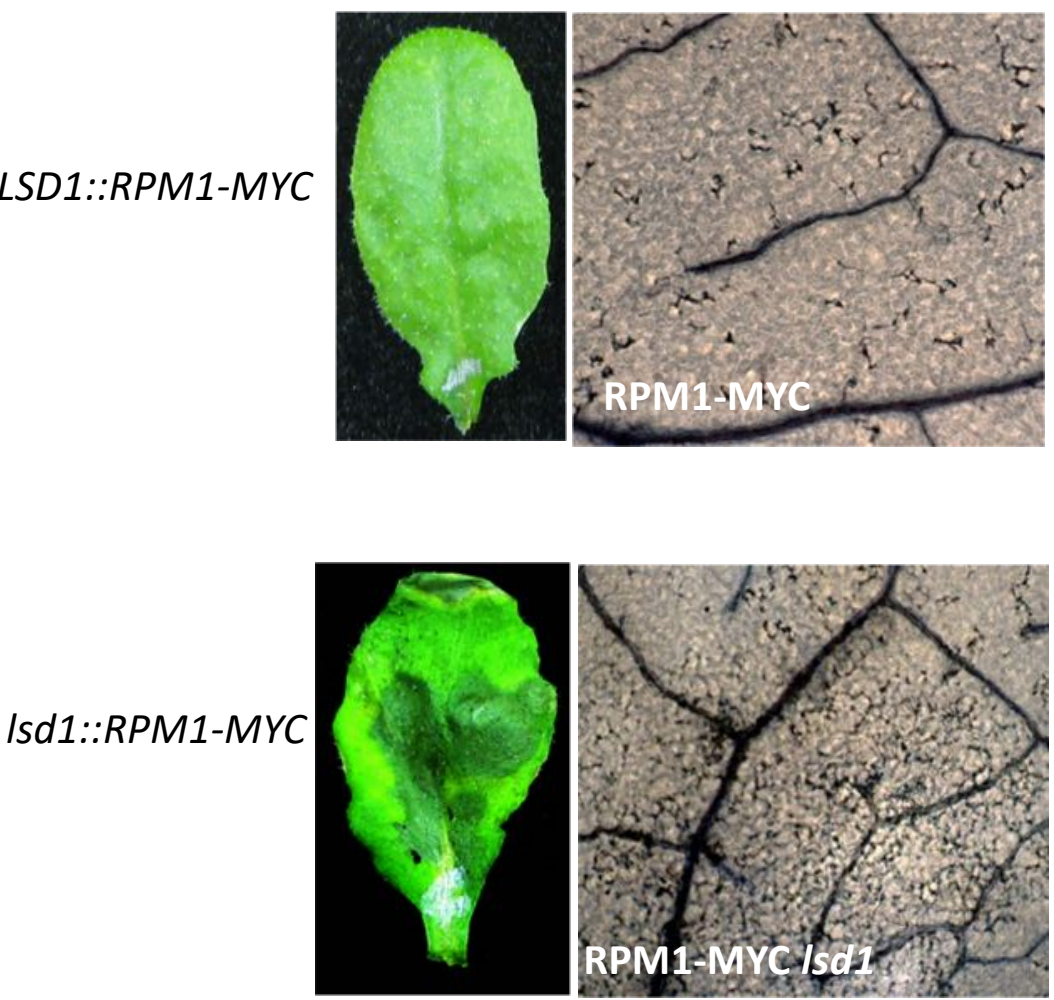

C
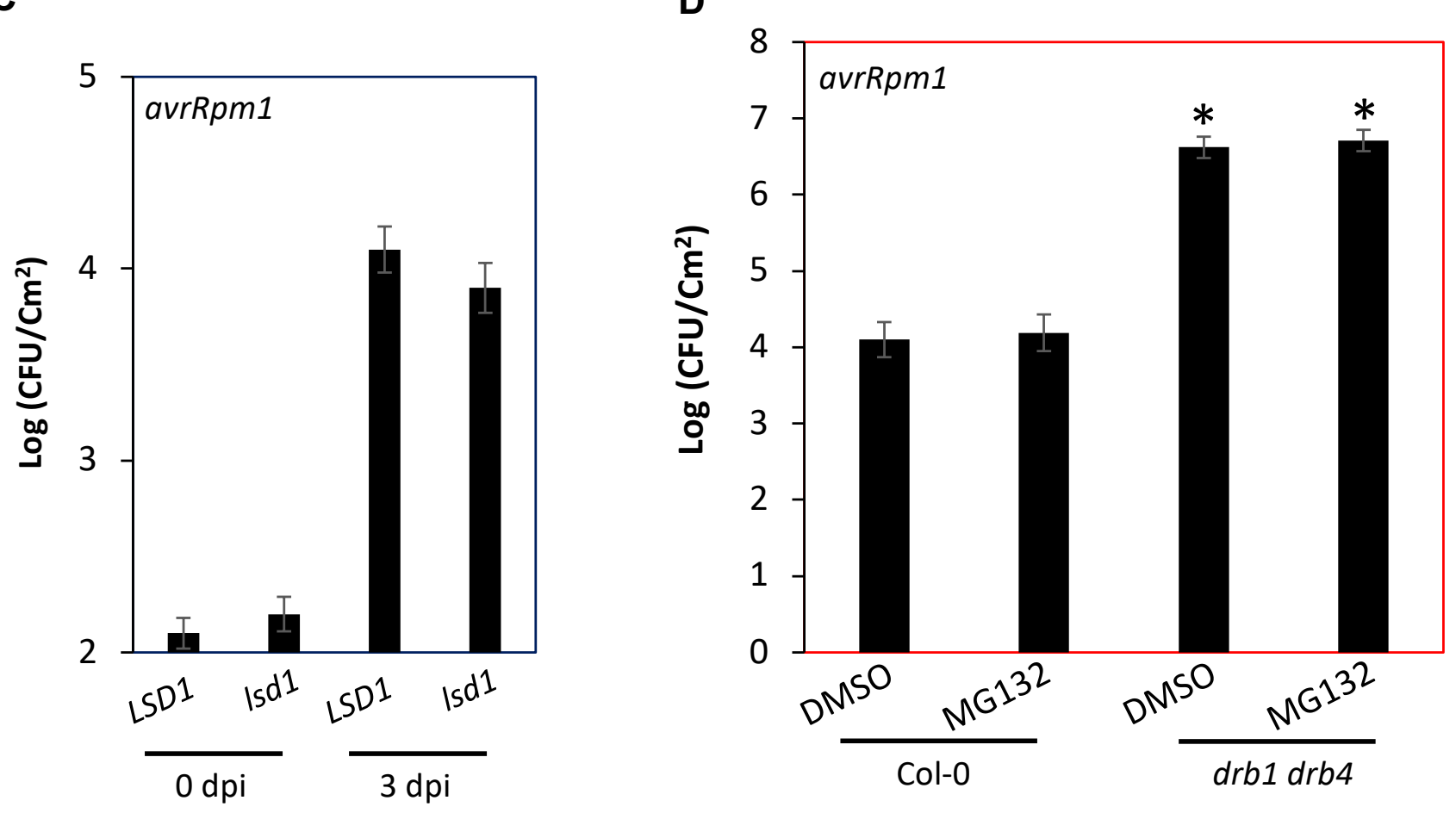
S. Figure 4
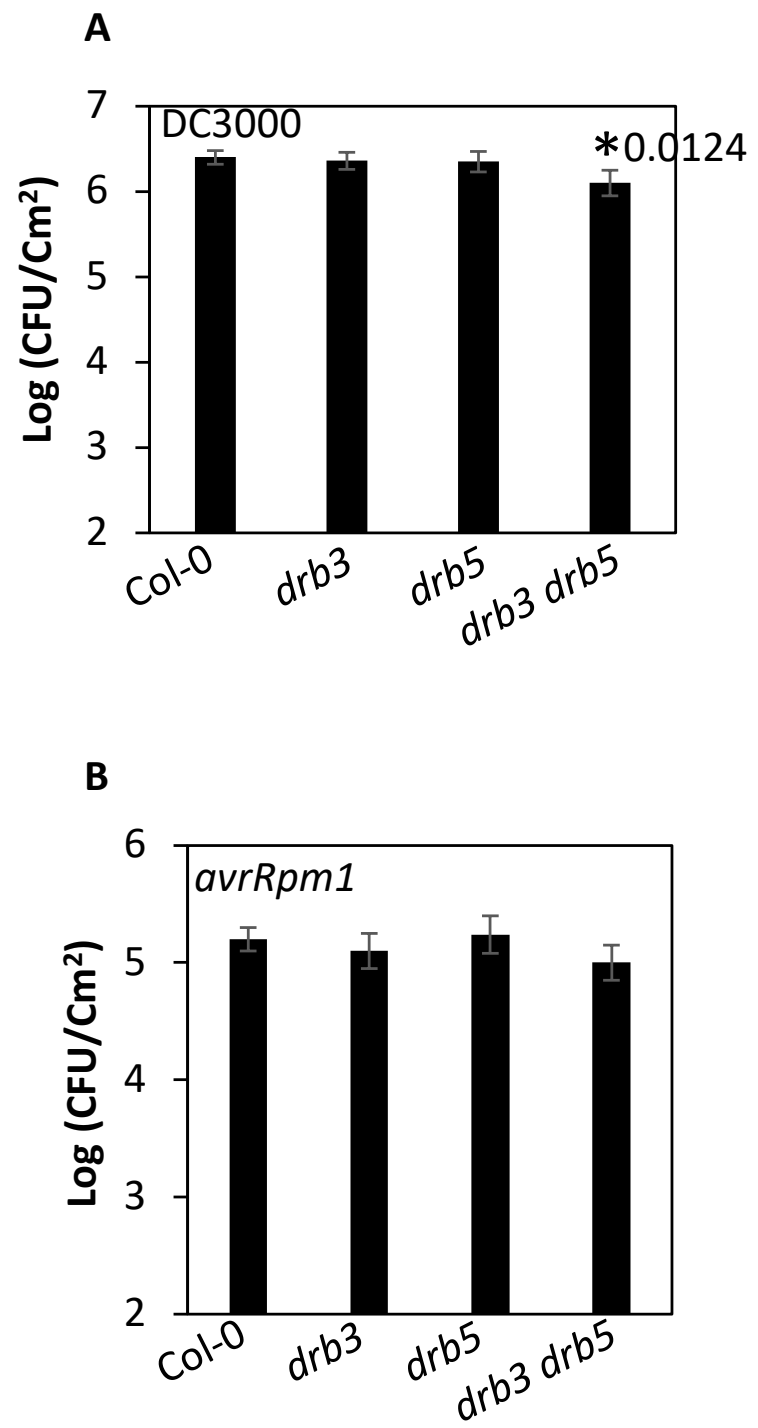
A

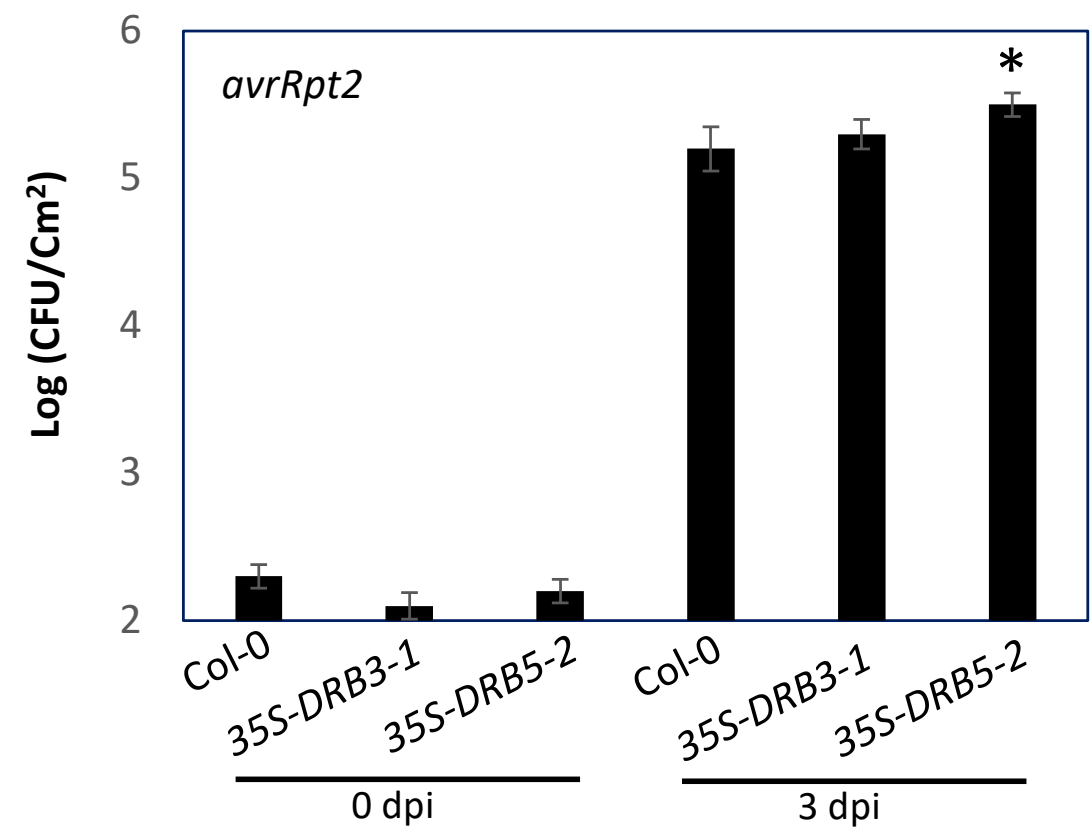

B

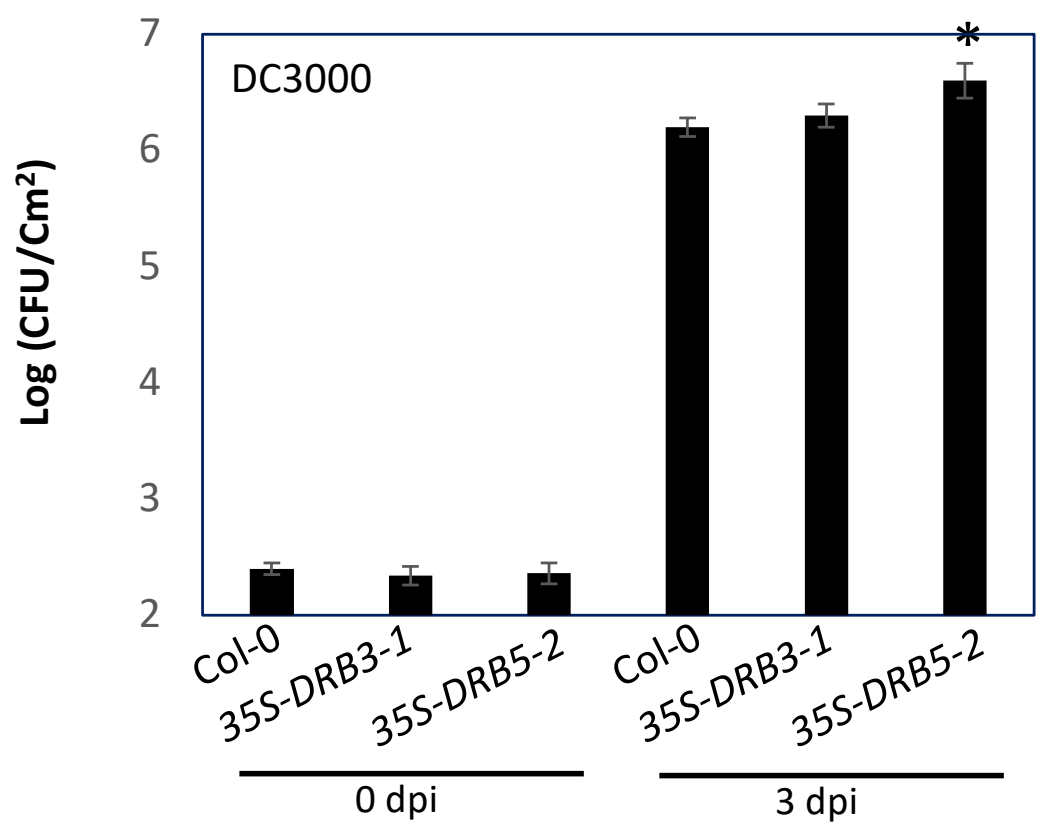

S. Figure 5 